\title{
The TR-BDF2 method for second order problems in structural mechanics
}

\author{
Luca Bonaventura $^{(1)}$, Macarena Gómez Mármol ${ }^{(2)}$ \\ July 27,2020 \\ (1) MOX - Modelling and Scientific Computing, \\ Dipartimento di Matematica "F. Brioschi", Politecnico di Milano \\ Via Bonardi 9, 20133 Milano, Italy \\ luca.bonaventura@polimi.it \\ (2) Departamento de Ecuaciones Diferenciales y Análisis Numérico, \\ Universidad de Sevilla \\ Apdo. de correos 1160, 41080 Sevilla, Spain \\ macarena@us.es
}

Keywords: Structural dynamics, highly oscillatory problems, Newmark method, Diagonally Implicit Runge Kutta methods, TR-BDF2 method.

AMS Subject Classification: 65L04, 65L06, 70-08, 74S99, 74H15 


\begin{abstract}
The application of the TR-BDF2 method to second order problems typical of structural mechanics and seismic engineering is discussed. A reformulation of this method is presented, that only requires the solution of algebraic systems of size equal to the number of displacement degrees of freedom. A linear analysis and numerical experiments on relevant benchmarks show that the TR-BDF2 method is superior in terms of accuracy and efficiency to the classical Newmark method and to its generalizations.
\end{abstract}




\section{Introduction}

The Newmark method [21] is an implicit time discretization technique for second order ordinary differential equations that is very widely used in structural dynamics applications, when the stiffness of the system causes standard explicit methods such as the StörmerVerlet method [11 to be inefficient. The so called Generalized $\alpha-$ methods [7] are extensions of the Newmark method using different time averaging parameters for different forcing terms. A full reference list and discussion of the properties of these methods are reported in [9], along with an analysis of their behaviour in the limit of arbitrarily large frequencies. Generalized $\alpha$-methods (shortly denoted as $\mathrm{G}(\alpha)$ methods in the following) introduce numerical dissipation in the approximation of the highest frequency modes of the solution, thus allowing to achieve unconditional stability and, in some regimes, to avoid overshoots, as discussed in [9]. However, in fully nonlinear regimes spurious oscillations may still be present. Furthermore, the stability and accuracy are dependent on the values of the numerical parameters that define the method, which have to be tuned for each specific application. For these reasons, alternatives to the $\mathrm{G}(\alpha)$ methods have been sought in 22], 23] in the class of Singly Diagonally Implicit Runge Kutta (SDIRK) and Rosenbrock methods, respectively. These investigations, which date back more than 25 years by now, do not seem to have changed the attitude of practitioners, who apparently have kept on using $\mathrm{G}(\alpha)$ methods for this kind of problems, apart from a limited number of exceptions, see e.g. [5], [6], [12], [13], [18], [20]. One possible reason of this preference is that, in the straightforward application of Runge-Kutta type methods to structural dynamics, the size of the system to be solved is twice that required by $\mathrm{G}(\alpha)$ methods.

In this work, we extend the analysis and comparison of [22] to the TR-BDF2 method [1], a second order accurate method whose remarkable accuracy, stability and efficiency properties have been fully analyzed in [16]. In particular, this L-stable method is endowed with an embedded third order method that permits effective time step adaptation. Unconditionally monotonic extensions of the TR-BDF2 method have been recently derived in [4] and its multirate version has been shown in [3] to be quite effective in reducing the computational cost of the time discretization for multirate hyperbolic problems. Furthermore, the combination of this method with high order, adaptive discontinuous finite element discretizations for model problems represented by first order equations in time has been shown in [25] to be extremely effective in reducing the computational cost of these high order methods. 
For these reasons, it is of interest to derive a form of this method that allows its application to structural mechanics problems. More specifically, we present a reformulation of the TR-BDF2 method for structural mechanics problems that only implies the solution of nonlinear systems of the same size as the number of displacement degrees of freedom. We also show how the velocity degrees of freedom do not have to be stored explicitly and can be recomputed whenever needed, thus avoiding excessive memory requirements for large scale applications. A similar reformulation was presented in 23 . for a two stage Rosenbrock method. An analysis of the accuracy and dissipation properties of the TR-BDF2 method is carried out in the linear regime, extending the classical analyses to consider also the approximation of the damping terms. Both this analysis and a number of numerical experiments on relevant benchmarks show that TR-BDF2 is superior in terms of accuracy and efficiency to the classical $\mathrm{G}(\alpha)$ methods.

The paper is organized as follows. In section 2 we show how the TR-BDF2 method can be applied to second order problems and reformulated so as to avoid solving systems of dimension larger than the number of displacement degrees of freedom, while at the same time not requiring extra storage of the velocity degrees of freedom. In section 3, the dissipation properties of the method are compared with those of the $\mathrm{G}(\alpha)$ methods. In section 4 results of several numerical simulations are presented, which highlight the accuracy and efficiency of the method for structural mechanics applications. Some conclusions and perspectives for future work are presented in section 5 .

\section{The TR-BDF2 method for second order problems}

We first consider the generic Cauchy problem $\mathbf{y}^{\prime}=\mathbf{f}(\mathbf{y}, t)$ on the time interval $t \in[0, T]$. Even though all the results presented in the following also hold for variable time steps, we present for simplicity the TR-BDF2 method as employing a constant time step $h=T / N$ and we follow in this presentation the notation and conventions in [4]. In its original formulation [1, the TR-BDF2 method is defined by the two stages:

$$
\begin{aligned}
& \mathbf{u}^{n \gamma}-\frac{\gamma h}{2} \mathbf{f}\left(\mathbf{u}^{n \gamma}, t_{n}+\gamma h\right)=\mathbf{u}^{n}+\frac{\gamma h}{2} \mathbf{f}\left(\mathbf{u}^{n}, t_{n}\right), \\
& \mathbf{u}^{n+1}-\gamma_{2} h \mathbf{f}\left(\mathbf{u}^{n+1}, t_{n+1}\right)=\left(1-\gamma_{3}\right) \mathbf{u}^{n}+\gamma_{3} \mathbf{u}^{n+\gamma} .
\end{aligned}
$$

Here, $\mathbf{u}^{n}$ denotes the numerical approximation of the solution at time 
level $t_{n}=n h, \gamma \in(0,1)$ is an implicitness parameter and $\gamma_{2}=(1-$ $\gamma) /(2-\gamma), \gamma_{3}=1 / \gamma(2-\gamma)$. The first stage of (1) is nothing but the application of the trapezoidal rule (or Crank-Nicolson method) over the interval $\left[t_{n}, t_{n}+\gamma h\right]$. The outcome of this stage is then used as input for the BDF2 implicit method. The resulting combination yields a method with several interesting accuracy and stability properties. A detailed analysis of these properties is given in [16], where it is also shown that TR-BDF2 is equivalent to a three stage Diagonally Implicit Runge Kutta (DIRK) method defined by the stages

$$
\begin{aligned}
\mathbf{k}_{1} & =\mathbf{f}\left(\mathbf{u}^{n}, t_{n}\right) \\
\mathbf{k}_{2} & =\mathbf{f}\left(\mathbf{u}^{n}+\frac{\gamma h}{2} \mathbf{k}_{1}+\frac{\gamma h}{2} \mathbf{k}_{2}, t_{n}+\gamma h\right) \\
\mathbf{k}_{3} & =\mathbf{f}\left(\mathbf{u}^{n}+\frac{\gamma_{3} h}{2} \mathbf{k}_{1}+\frac{\gamma_{3} h}{2} \mathbf{k}_{2}+\gamma_{2} h \mathbf{k}_{3}, t_{n+1}\right) \\
\mathbf{u}^{n+1} & =\mathbf{u}^{n}+h\left(\frac{\gamma_{3}}{2} \mathbf{k}_{1}+\frac{\gamma_{3}}{2} \mathbf{k}_{2}+\gamma_{2} \mathbf{k}_{3}\right) .
\end{aligned}
$$

Notice that this method is not Singly Diagonally Implicit Runge Kutta (SDIRK), due to the fully explicit first stage. As shown in [16], the TR-BDF2 method is second order accurate and A-stable for any value of $\gamma$. Furthermore, for $\gamma=2-\sqrt{2}$ it is also L-stable. Therefore, with this coefficient value it can be safely applied to problems with eigenvalues whose imaginary part is large, such as typically arise from the discretization of highly oscillatory second order problems. This is not the case for the standard trapezoidal rule (or Crank-Nicolson) implicit method, whose linear stability region is exactly bounded by the imaginary axis. Recently, the method has been applied in [25], 24] to high order accuracy spatial discretization of wave propagation phenomena written as systems of first order in time. Its monotonicity properties have also been studied in [4], where it was shown that an unconditionally monotonic extension of the method can be derived and that the method does not suffer from order reduction when applied to stiff systems. Several specific variants of the method for nonlinear problems have been proposed and analyzed in [8], while an analysis of a multirate implicit method based on TR-BDF2 has been presented in $[3$.

We now focus on systems of second order ordinary differential equations. In particular, as common in structural mechanics applications, we will consider systems of the form

$$
\mathbf{M} \mathbf{y}^{\prime \prime}=-\mathbf{C y}^{\prime}-\mathbf{K} \mathbf{y}+\mathbf{g}(\mathbf{y})+\mathbf{z}(t)
$$

with the initial conditions $\mathbf{y}(0)=\mathbf{y}_{0} \quad \mathbf{y}^{\prime}(0)=\mathbf{v}_{0}$. Here, $\mathbf{M}$ denotes a symmetric and positive definite mass matrix, $\mathbf{K}$ denotes the stiffness 
matrix, which is also assumed to be symmetric and positive definite, while the matrix $\mathbf{C}$ represents friction terms.

In order to introduce the application of the TR-BDF2 method to this kind of problems, system (3) is rewritten as a first order system letting $\mathbf{v}=\mathbf{y}^{\prime}$ and setting $\mathbf{\Gamma}=\mathbf{M}^{-1} \mathbf{C}, \mathbf{B}=\mathbf{M}^{-1} \mathbf{K}$, and $\mathbf{f}(\mathbf{y})=$ $\mathbf{M}^{-1} \mathbf{g}(\mathbf{y}), \mathbf{s}(t)=\mathbf{M}^{-1} \mathbf{z}(t)$, so as to obtain

$$
\begin{aligned}
& \mathbf{y}^{\prime}=\mathbf{v} \\
& \mathbf{v}^{\prime}=-\mathbf{\Gamma} \mathbf{v}-\mathbf{B y}+\mathbf{f}(\mathbf{y})+\mathbf{s}(t) .
\end{aligned}
$$

We now apply method (1) to problem (4). Denoting by $\mathbf{u}^{n}, \mathbf{w}^{n}$, respectively, the numerical approximations of $\mathbf{y}, \mathbf{v}$ at time level $n$, the trapezoidal rule stage can be written as

$$
\begin{aligned}
\mathbf{u}^{n+\gamma} & =\mathbf{u}^{n}+\frac{\gamma h}{2} \mathbf{w}^{n}+\frac{\gamma h}{2} \mathbf{w}^{n+\gamma} \\
\mathbf{w}^{n+\gamma} & +\frac{\gamma h}{2} \boldsymbol{\Gamma} \mathbf{w}^{n+\gamma}+\frac{\gamma h}{2} \mathbf{B} \mathbf{u}^{n+\gamma}-\frac{\gamma h}{2} \mathbf{f}\left(\mathbf{u}^{n+\gamma}\right) \\
& =\mathbf{w}^{n}-\frac{\gamma h}{2} \boldsymbol{\Gamma} \mathbf{w}^{n}-\frac{\gamma h}{2} \mathbf{B} \mathbf{u}^{n}+\frac{\gamma h}{2} \mathbf{f}\left(\mathbf{u}^{n}\right)+\frac{\gamma h}{2} \overline{\mathbf{s}},
\end{aligned}
$$

where we have set $\overline{\mathbf{s}}=\mathbf{s}\left(t^{n+\gamma}\right)+\mathbf{s}\left(t^{n}\right)$. The BDF2 stage yields instead

$$
\begin{aligned}
\mathbf{u}^{n+1} & =\gamma_{2} h \mathbf{w}^{n+1}+\left(1-\gamma_{3}\right) \mathbf{u}^{n}+\gamma_{3} \mathbf{u}^{n+\gamma} \\
\mathbf{w}^{n+1} & +\gamma_{2} h \boldsymbol{\Gamma} \mathbf{w}^{n+1}+\gamma_{2} h \mathbf{B} \mathbf{u}^{n+1}-\gamma_{2} h \mathbf{f}\left(\mathbf{u}^{n+1}\right) \\
& =\left(1-\gamma_{3}\right) \mathbf{w}^{n}+\gamma_{3} \mathbf{w}^{n+\gamma}+\gamma_{2} h \mathbf{s}^{n+1} .
\end{aligned}
$$

Along the lines of what is done for the Newmark and G( $\alpha)$ methods, each of the two stages is now rewritten in terms of a single implicit step for $\mathbf{u}^{n+\gamma}, \mathbf{u}^{n+1}$, respectively. This amounts to

$$
\begin{aligned}
\mathbf{w}^{n+\gamma} & =2\left(\mathbf{u}^{n+\gamma}-\mathbf{u}^{n}-\gamma h \mathbf{w}^{n} / 2\right) / \gamma h \\
\mathbf{A}_{1} \mathbf{u}^{n+\gamma} & -\frac{\gamma^{2} h^{2}}{4} \mathbf{f}\left(\mathbf{u}^{n+\gamma}\right)=\mathbf{b}_{1} \\
\mathbf{w}^{n+1} & =\left[\mathbf{u}^{n+1}-\left(1-\gamma_{3}\right) \mathbf{u}^{n}-\gamma_{3} \mathbf{u}^{n+\gamma}\right] / \gamma_{2} h \\
\mathbf{A}_{2} \mathbf{u}^{n+1} & -\gamma_{2}^{2} h^{2} \mathbf{f}\left(\mathbf{u}^{n+1}\right)=\mathbf{b}_{2}
\end{aligned}
$$


where we have now set

$$
\begin{aligned}
\mathbf{A}_{1} & =\mathbf{I}+\frac{\gamma h}{2} \boldsymbol{\Gamma}+\frac{\gamma^{2} h^{2}}{4} \mathbf{B} \\
\mathbf{A}_{2} & =\mathbf{I}+\gamma_{2} h \boldsymbol{\Gamma}+\gamma_{2}^{2} h^{2} \mathbf{B} \\
\mathbf{b}_{1} & =\left(\mathbf{I}+\frac{\gamma h}{2} \boldsymbol{\Gamma}-\frac{\gamma^{2} h^{2}}{4} \mathbf{B}\right) \mathbf{u}^{n} \\
& +\frac{\gamma^{2} h^{2}}{4} \mathbf{f}\left(\mathbf{u}^{n}\right)+\frac{\gamma^{2} h^{2}}{4} \overline{\mathbf{s}}+\gamma h \mathbf{w}^{n} \\
\mathbf{b}_{2} & =\gamma_{2}^{2} h^{2} \mathbf{s}^{n+1}+\gamma_{2}\left(1-\gamma_{3}\right) h \mathbf{w}^{n}+\gamma_{2} \gamma_{3} h \mathbf{w}^{n+\gamma} \\
& +\left(1-\gamma_{3}\right)\left(\mathbf{I}+\gamma_{2} h \boldsymbol{\Gamma}\right) \mathbf{u}^{n}+\gamma_{3}\left(\mathbf{I}+\gamma_{2} h \boldsymbol{\Gamma}\right) \mathbf{u}^{n+\gamma} .
\end{aligned}
$$

Fixing from now on the value $\gamma=2-\sqrt{2}$, so as to achieve L-stability, one obtains $\gamma_{2}=\gamma / 2$, so that $\mathbf{A}_{1}=\mathbf{A}_{2}=\mathbf{A}$. Notice that this formulation has a number of advantages. First, it allows in practice to avoid doubling the degrees of freedom of the discrete problem, even though a method for first order problems is employed. For the first time step, the value of $\mathbf{w}^{n}$ would be recovered from the initial datum, while formula (11) allows the reconstruction of the same term at subsequent time levels. Furthermore, both stages of the method are defined in terms of the same matrix. In order to improve the efficiency of the algebraic solvers, both equations can also be rewritten in terms of the increments

$$
\delta \mathbf{u}^{n+\gamma}=\mathbf{u}^{n+\gamma}-\mathbf{u}^{n} \quad \delta \mathbf{u}^{n+1}=\mathbf{u}^{n+1}-\mathbf{u}^{n+\gamma},
$$

so that the scheme can be rewritten as

$$
\begin{aligned}
& \mathbf{A} \delta \mathbf{u}^{n+\gamma}-\frac{\gamma^{2} h^{2}}{4} \mathbf{f}\left(\mathbf{u}^{n}+\delta \mathbf{u}^{n+\gamma}\right)=\mathbf{b}_{1}-\mathbf{A} \mathbf{u}^{n} \\
& \mathbf{A} \delta \mathbf{u}^{n+1}-\frac{\gamma^{2} h^{2}}{4} \mathbf{f}\left(\mathbf{u}^{n+\gamma}+\delta \mathbf{u}^{n+1}\right)=\mathbf{b}_{2}-\mathbf{A} \mathbf{u}^{n+\gamma} .
\end{aligned}
$$

Finally, it is easy to notice that the mass matrix inversion is not necessary in practice. Indeed, each of the previous equations can be multiplied by $\mathbf{M}$, thus yielding the two nonlinear systems

$$
\begin{aligned}
& \mathbf{A} \delta \mathbf{u}^{n+\gamma}-\frac{\gamma^{2} h^{2}}{4} \mathbf{g}\left(\mathbf{u}^{n}+\delta \mathbf{u}^{n+\gamma}\right)=\tilde{\mathbf{b}}_{1} \\
& \mathbf{A} \delta \mathbf{u}^{n+1}-\frac{\gamma^{2} h^{2}}{4} \mathbf{g}\left(\mathbf{u}^{n+\gamma}+\delta \mathbf{u}^{n+1}\right)=\tilde{\mathbf{b}}_{2}
\end{aligned}
$$


where now $\mathbf{A}$ has been redefined as $\mathbf{A}=\mathbf{M}+\gamma h \mathbf{C} / 2+\gamma^{2} h^{2} \mathbf{K} / 4$ and the corresponding right hand sides as

$$
\begin{aligned}
\tilde{\mathbf{b}}_{1} & =\left(\mathbf{M}+\frac{\gamma h}{2} \mathbf{C}-\frac{\gamma^{2} h^{2}}{4} \mathbf{K}\right) \mathbf{u}^{n} \\
& +\frac{\gamma^{2} h^{2}}{4} \mathbf{g}\left(\mathbf{u}^{n}\right)+\frac{\gamma^{2} h^{2}}{4} \overline{\mathbf{z}}+\gamma h \mathbf{w}^{n}-\mathbf{A} \mathbf{u}^{n} \\
\tilde{\mathbf{b}}_{2} & =\gamma_{2}^{2} h^{2} \mathbf{z}^{n+1}+\gamma_{2}\left(1-\gamma_{3}\right) h \mathbf{M w}^{n}+\gamma_{2} \gamma_{3} h \mathbf{M} \mathbf{w}^{n+\gamma} \\
& +\left(1-\gamma_{3}\right)\left(\mathbf{M}+\gamma_{2} h \mathbf{C}\right) \mathbf{u}^{n}+\gamma_{3}\left(\mathbf{M}+\gamma_{2} h \mathbf{C}\right) \mathbf{u}^{n+\gamma}-\mathbf{A} \mathbf{u}^{n+\gamma}
\end{aligned}
$$

For completeness, we also present the classical Newmark method and its $\mathrm{G}(\alpha)$ generalizations. Following [9], we introduce discrete approximations $\mathbf{u}^{n}, \mathbf{w}^{n}, \mathbf{a}^{n}$ of the continuous displacement, velocity and acceleration values, respectively:

$$
\begin{aligned}
& \mathbf{u}^{n+1}=\mathbf{u}^{n}+h \mathbf{w}^{n}+h^{2}\left[\left(\beta-\frac{1}{2}\right) \mathbf{a}^{n}+\beta \mathbf{a}^{n+1}\right] \\
& \mathbf{w}^{n+1}=\mathbf{w}^{n}+h\left[\left(1-\gamma_{N}\right) \mathbf{a}^{n}+\gamma_{N} \mathbf{a}^{n+1}\right] \\
& \mathbf{M a}^{n+1-\alpha_{m}}+\mathbf{C w}^{n+1-\alpha_{f}}+\mathbf{K} \mathbf{u}^{n+1-\alpha_{f}} \\
& =\mathbf{g}\left(\mathbf{u}^{n+1-\alpha_{f}}\right)+\mathbf{z}^{n+1-\alpha_{f}},
\end{aligned}
$$

where for a generic variable $\phi$ one has $\phi^{n+1-\alpha}=(1-\alpha) \phi^{n+1}+\alpha \phi^{n}$. where $\gamma_{N}, \beta, \alpha_{m}, \alpha_{f}$ are specific averaging parameters. Notice that different definitions of the $\alpha$ averaging can also be employed and that several relationships between the values of the parameters $\gamma_{N}, \beta, \alpha_{m}, \alpha_{f}$ must be respected for the method to be second order consistent in the displacement and unconditionally stable. We refer again to [9] for a complete discussion of these issues and of a simple strategy to link the values of these parameters to that of the effective dissipation rate of the highest frequencies, denoted by $\rho_{\infty}$. Here, we just summarize the key results, which are reported in the following expression

$$
\beta=\frac{1}{\left(1+\rho_{\infty}\right)^{2}} \quad \gamma_{N}=\frac{1}{2} \frac{3-\rho_{\infty}}{1+\rho_{\infty}}
$$

for the parameters $\beta, \gamma_{N}$ and

$$
\alpha_{f}=\frac{2 \rho_{\infty}-1}{1+\rho_{\infty}} \quad \alpha_{m}=\frac{\rho_{\infty}}{1+\rho_{\infty}}
$$

for the parameters that define the $\mathrm{G}(\alpha)$ method introduced in [7], which we consider in the following as example of the broader $\mathrm{G}(\alpha)$ class. 


\section{Analysis of the TR-BDF2 method}

In [22], several SDIRK methods were compared to some $\mathrm{G}(\alpha)$ methods with respect to their dissipation properties. The behaviour of the TR-BDF2 method is very similar to the two stage SDIRK method analyzed in Section 2.2 of [22]. However, the analysis will be repeated here for convenience and extended to the case in which also dissipative terms are taken into account. More specifically, we will consider the methods introduced in section 2 as applied to the linear scalar problem

$$
y^{\prime \prime}=-\frac{c}{m} y^{\prime}-\frac{k}{m} y,
$$

where $k, m>0, c \geq 0$. We will only consider the case in which $c^{2}<$ $4 k m$, for which it is well known that the analytic solution has the form

$$
y(t)=A_{+} \exp \left(\alpha_{+} t\right)+A_{-} \exp \left(\alpha_{-} t\right),
$$

where one has

$$
\alpha_{ \pm}=-\tilde{\zeta} \pm i \tilde{\omega} \quad \text { with } \quad \tilde{\zeta}=c / 2 m \geq 0, \quad \tilde{\omega}=\sqrt{k / m-(c / 2 m)^{2}}>0 .
$$

As a consequence, one can write for this case the exact evolution operator

$$
\mathbf{y}^{n+1}=\mathbf{E y}^{n},
$$

where we have set $\mathbf{y}^{n}=\left[y\left(t^{n}\right), h y^{\prime}\left(t^{n}\right)\right]^{T}, \zeta=h \tilde{\zeta}, \omega=h \tilde{\omega}$ and

$$
\mathbf{E}=\left[\begin{array}{cc}
\left(\cos \omega+\frac{\zeta}{\omega} \sin \omega\right) \mathrm{e}^{-\zeta} & \frac{\sin \omega}{\omega} \mathrm{e}^{-\zeta} \\
-\left(\zeta^{2}+\omega^{2}\right) \frac{\sin \omega}{\omega} \mathrm{e}^{-\zeta} & \left(\cos \omega-\frac{\zeta}{\omega} \sin \omega\right) \mathrm{e}^{-\zeta}
\end{array}\right]
$$

Notice that the velocity variable has been rescaled (see e.g. [2]), so as to obtain an evolution operator which only includes dimensionally homogeneous quantities. Defining then $\mathbf{x}^{n}=\left[u^{n}, z^{n}\right]^{T}$, where we set $z^{n}=h w^{n}$, the discrete evolution operator corresponding to the TRBDF2 method can be reconstructed from the specific forms of the TR and BDF2 stages, which can be written as

$$
\mathbf{S}_{1} \mathbf{x}^{n+\gamma}=\mathbf{T}_{1} \mathbf{x}^{n} \quad \mathbf{S}_{2} \mathbf{x}^{n+1}=\mathbf{T}_{2} \mathbf{x}^{n+\gamma}+\mathbf{U}_{2} \mathbf{x}^{n},
$$


where one defines

$$
\begin{aligned}
& \mathbf{S}_{1}=\left[\begin{array}{cc}
\left(1+\gamma \zeta+\left(\omega^{2}+\zeta^{2}\right) \frac{\gamma^{2}}{4}\right) & 0 \\
1 & -\frac{\gamma}{2}
\end{array}\right] \\
& \mathbf{T}_{1}=\left[\begin{array}{cc}
\left(1+\gamma \zeta-\left(\omega^{2}+\zeta^{2}\right) \frac{\gamma^{2}}{4}\right) & \gamma \\
1 & \frac{\gamma}{2}
\end{array}\right] \\
& \mathbf{S}_{2}=\left[\begin{array}{cc}
\left(1+\gamma \zeta+\left(\omega^{2}+\zeta^{2}\right) \frac{\gamma^{2}}{4}\right) & 0 \\
1 & -\frac{\gamma}{2}
\end{array}\right] \\
& \mathbf{T}_{2}=\left[\begin{array}{cc}
\frac{1}{\gamma(2-\gamma)}\left(1+\frac{\gamma}{2} \zeta\right) & \frac{1}{2(2-\gamma)} \\
\frac{1}{\gamma(2-\gamma)} & 0
\end{array}\right] \\
& \mathbf{U}_{2}=\left[\begin{array}{cc}
-\frac{(1-\gamma)^{2}}{\gamma(2-\gamma)}\left(1+\frac{\gamma}{2} \zeta\right) & -\frac{(1-\gamma)^{2}}{2(2-\gamma)} \\
-\frac{(1-\gamma)^{2}}{\gamma(2-\gamma)} & 0
\end{array}\right] .
\end{aligned}
$$

By straightforward algebraic manipulations, one obtains

$$
\mathbf{x}^{n+1}=\mathbf{S}_{2}^{-1}\left[\mathbf{T}_{2}\left(\mathbf{S}_{2}^{-1} \mathbf{T}_{2}\right) \mathbf{U}_{2}\right] \mathbf{x}^{n},
$$

which can then be compared to 27) in order to assess the properties of the TR-BDF2 method. A similar comparison can be carried out for the $\mathrm{G}(\alpha)$ methods, taking into account that these methods also employ an approximation of the second derivative. Therefore, one has to consider the exact evolution operator derived from the relationship

$$
\left[\begin{array}{ll}
\mathbf{I} & 0 \\
\boldsymbol{\xi} & 1
\end{array}\right]\left[\begin{array}{c}
\mathbf{y}^{n+1} \\
h^{2} y^{\prime \prime}\left(t^{n+1}\right)
\end{array}\right]=\left[\begin{array}{ll}
\mathbf{E} & 0 \\
\mathbf{0} & 0
\end{array}\right]\left[\begin{array}{c}
\mathbf{y}^{n} \\
h^{2} y^{\prime \prime}\left(t^{n}\right)
\end{array}\right],
$$

where $\boldsymbol{\xi}=\left[\omega^{2}+\zeta^{2}, \zeta\right]^{T}$ and equation (26) has been used to introduce the relationship between acceleration, velocity and displacement values. Analogously, for the generic $\mathrm{G}(\alpha)$ method one has

$$
\begin{aligned}
& {\left[\begin{array}{ccc}
1 & 0 & -\beta h^{2} \\
0 & 1 & -\gamma_{N} \\
\left(1-\alpha_{f}\right) \omega^{2} & \left(1-\alpha_{f}\right) \zeta & \left(1-\alpha_{m}\right)
\end{array}\right]\left[\begin{array}{c}
u^{n+1} \\
h w^{n+1} \\
h^{2} a^{n+1}
\end{array}\right]} \\
& =\left[\begin{array}{ccc}
1 & 1 & (\beta-1 / 2) \\
0 & 1 & \left(1-\gamma_{N}\right) \\
\alpha_{f} \omega^{2} & \alpha_{f} \zeta & \alpha_{m}
\end{array}\right]\left[\begin{array}{c}
u^{n} \\
h w^{n} \\
h^{2} a^{n}
\end{array}\right],
\end{aligned}
$$

so that the comparison is between the exact operator

$$
\left[\begin{array}{ll}
\mathbf{I} & 0 \\
\boldsymbol{\xi} & 1
\end{array}\right]^{-1}\left[\begin{array}{ll}
\mathbf{E} & 0 \\
\mathbf{0} & 0
\end{array}\right]
$$


and the approximate operator

$$
\left[\begin{array}{ccc}
1 & 0 & -\beta h^{2} \\
0 & 1 & -\gamma_{N} \\
\left(1-\alpha_{f}\right) \omega^{2} & \left(1-\alpha_{f}\right) \zeta & \left(1-\alpha_{m}\right)
\end{array}\right]^{-1}\left[\begin{array}{ccc}
1 & 1 & (\beta-1 / 2) \\
0 & 1 & \left(1-\gamma_{N}\right) \\
\alpha_{f} \omega^{2} & \alpha_{f} \zeta & \alpha_{m}
\end{array}\right]
$$

The ratios of the spectral norms of the discrete evolution operators to that of the exact evolution operator are reported for different methods in Figure 1 as a function of $(\zeta, \omega)$, on the domain $(\zeta, \omega) \in[-1,0] \times[0,10]$. Notice that these quantities are symmetric with respect to the $\omega=0$ axis. The corresponding relative errors in the spectral norm with respect to the exact evolution operators are reported in Figure 2, In particular, the TR-BDF2 method, the Newmark method with three different values of the damping parameters and the $\mathrm{G}(\alpha)$ method introduced in [7] (denoted by $\mathrm{CH}-\alpha$ ) with two different values of the damping parameters are compared in this way.

It can be observed that the TR-BDF2 method introduces significantly less numerical damping in the ranges corresponding to frequencies that need to be accurately resolved, while damping is more effective on the highest frequencies. Furthermore, the TR-BDF2 method also introduces a much smaller error than its counterparts in the same frequency ranges. These findings, which could have been obtained in the undamped case with an analysis analogous to that presented in 22, are also true if damping is added, thus confirming the advantages of the TR-BDF2 method for realistic applications including possibly stiff dissipative terms. 


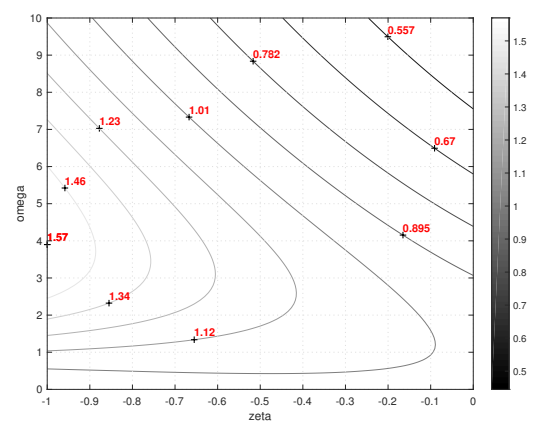

a)
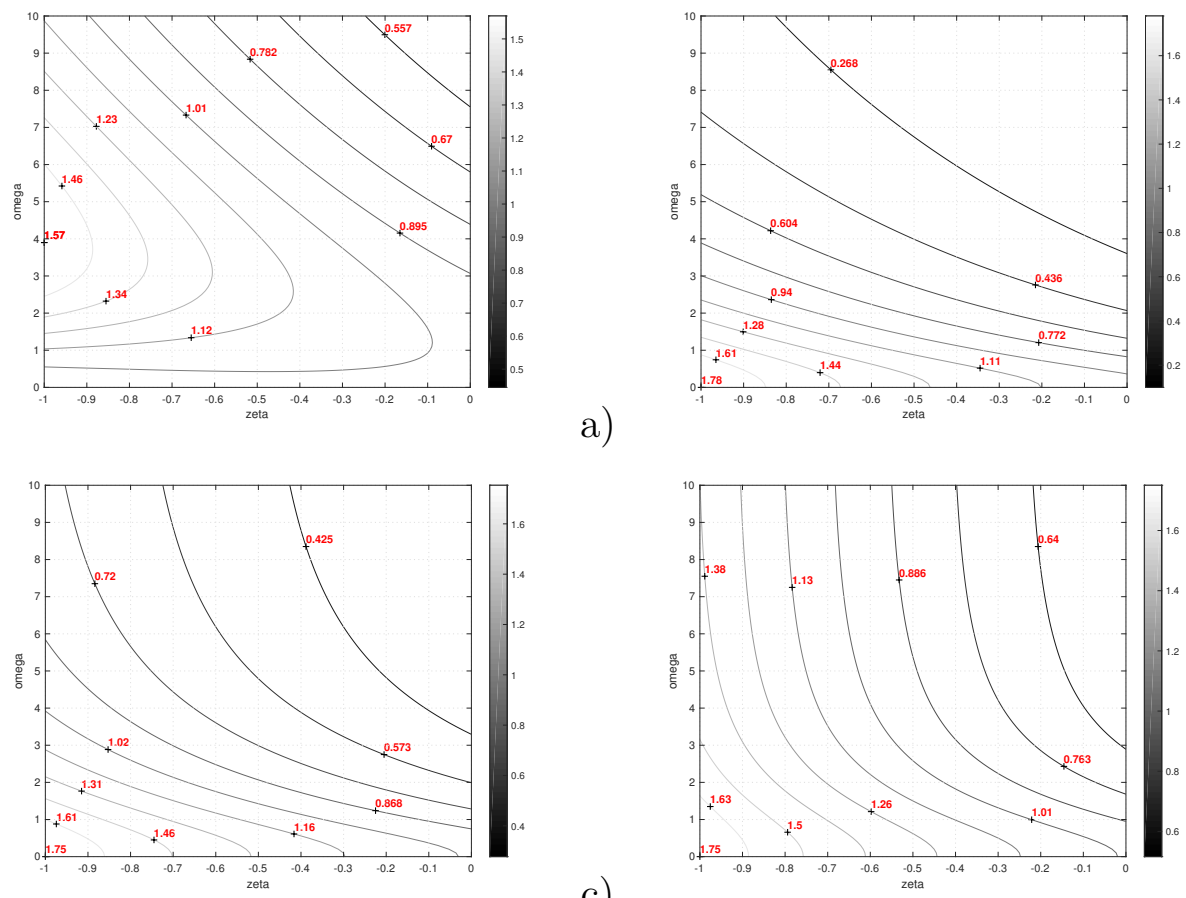

c)

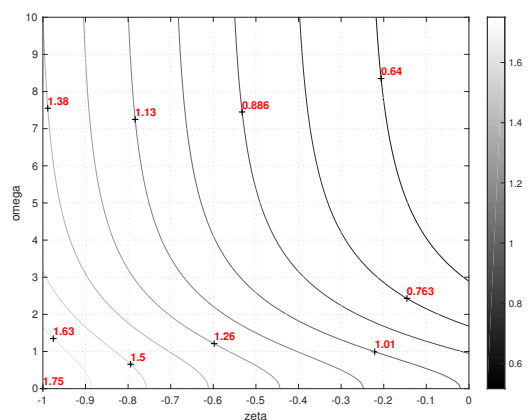

b)

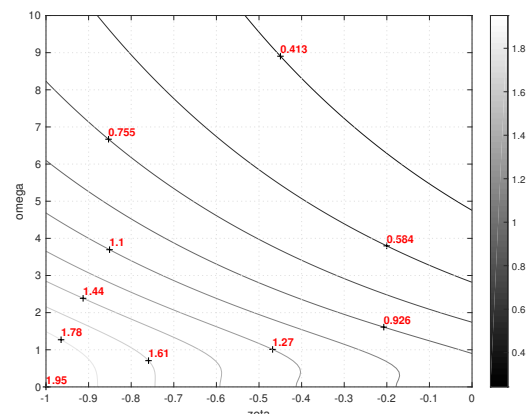

e)

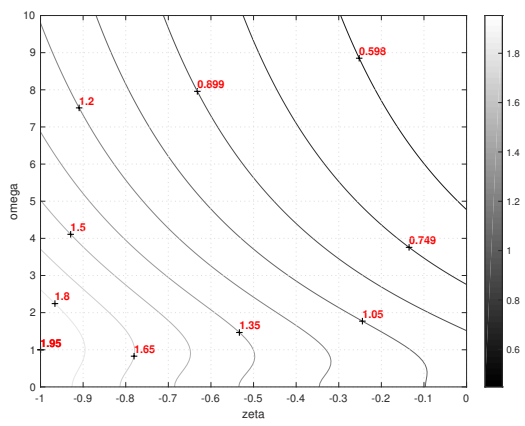

d)

f)

Figure 1: Ratio of the spectral norm of the discrete evolution operators to that of the exact evolution operator for a) TR-BDF2 method, b) the Newmark method with $\left.\rho_{\infty}=0, \mathrm{c}\right)$ the Newmark method with $\rho_{\infty}=0.25, \mathrm{~d}$ ) the Newmark method with $\rho_{\infty}=0.5$ e) the CH- $\alpha$ method with $\rho_{\infty}=0$ e) the $\mathrm{CH}-\alpha$ method with $\rho_{\infty}=0.25$. 

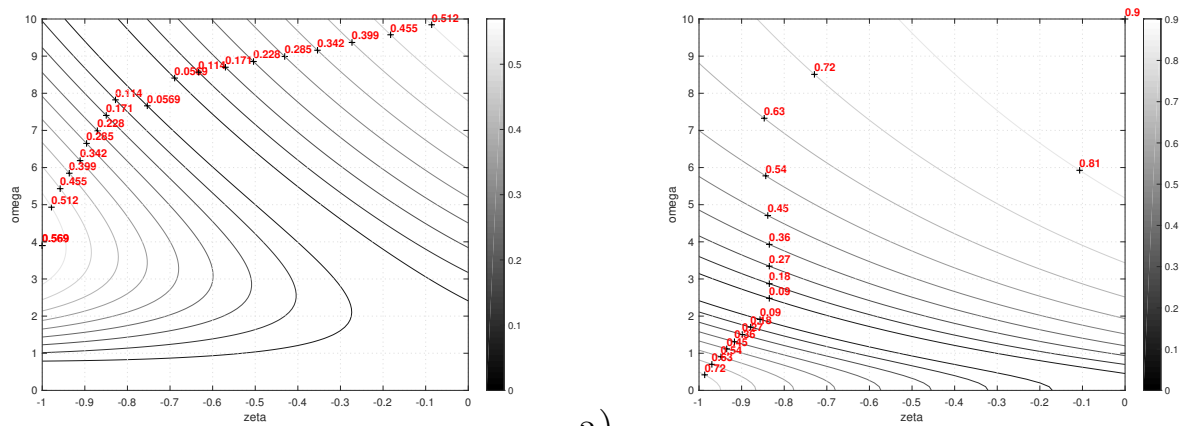

a)

b)

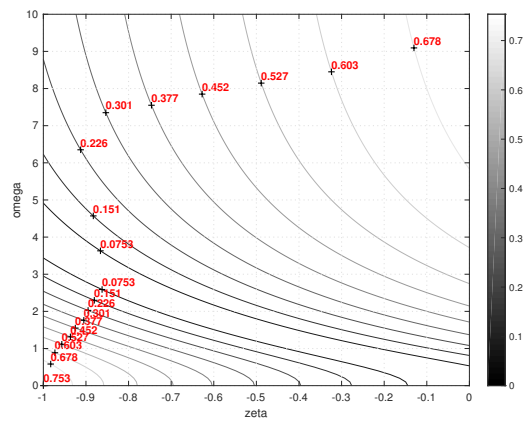

c)

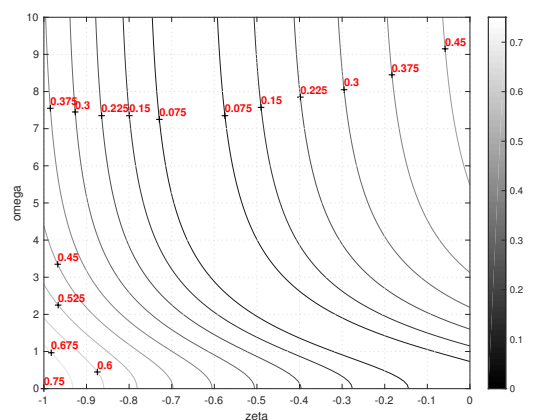

d)

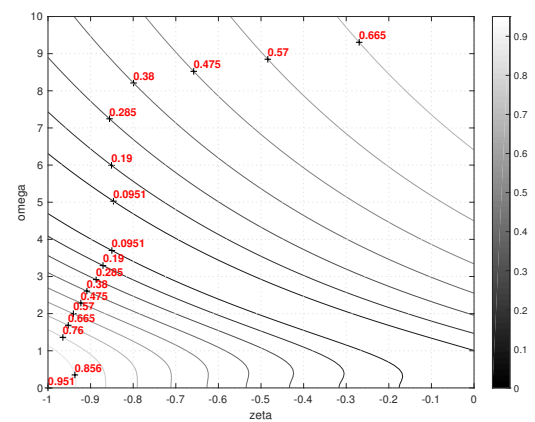

e)

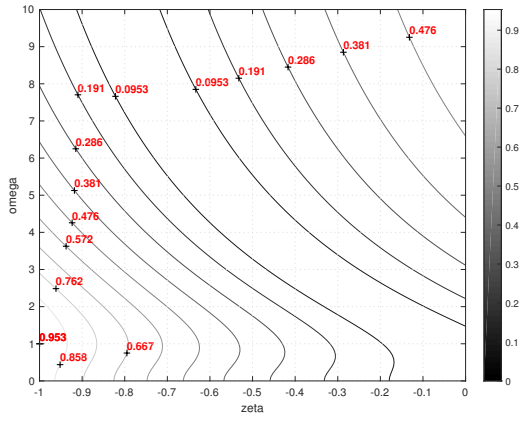

f)

Figure 2: Relative error in the spectral norm with respect to the exact evolution operator for a) TR-BDF2 method b) the Newmark method with $\rho_{\infty}=0$ c) the Newmark method with $\rho_{\infty}=0.25 \mathrm{~d}$ ) the Newmark method with $\rho_{\infty}=0.5$ e) the $\mathrm{CH}-\alpha$ method with $\rho_{\infty}=0$ e) the $\mathrm{CH}-\alpha$ method with $\rho_{\infty}=0.25$. 


\section{Numerical experiments}

A number of numerical experiments have been carried out in order to assess the accuracy and efficiency of the TR-BDF2 method for applications to structural mechanics and to compare it with that of the $\mathrm{G}(\alpha)$ methods. We first consider one of the nonlinear numerical benchmarks discussed in [9]. Then, time discretizations of the wave equation are considered, in cases which are representative of possible applications to structural mechanics and seismic engineering. For all the problems concerning the discretization of a wave equation, we have used a finite element spatial discretization and our computations have been performed with the free software FreeFem ++ [14, computing the errors in the space-time norms

$$
\begin{gathered}
\|u\|_{L^{\infty}\left(L^{2}\right)}=\max _{n}\left\|u\left(t^{n}, \cdot\right)\right\|_{L^{2}(\Omega)} \\
\|u\|_{L^{2}\left(H^{1}\right)}^{2}=\sum_{n}\left\|u\left(t^{N}, \cdot\right)\right\|_{H^{1}(\Omega)}^{2} \Delta t \\
\|u\|_{L^{\infty}\left(L^{\infty}\right)}=\max _{n}\left\|u\left(t^{n}, \cdot\right)\right\|_{L^{\infty}(\Omega)},
\end{gathered}
$$

where $t^{n}$ are the time levels used by the time discretization on $[0, T]$.

\subsection{Nonlinear system with 2 degrees of free- dom}

In a first numerical experiment, we consider the strongly nonlinear system with two degrees of freedom

$$
\left[\begin{array}{l}
y_{1} \\
y_{2}
\end{array}\right]^{\prime \prime}=-\left[\begin{array}{c}
10^{4} y_{1}\left(1+10^{4} y_{1}^{2}\right)-\tanh \left(y_{2}-y_{1}\right) \\
\tanh \left(y_{2}-y_{1}\right)
\end{array}\right]
$$

with the initial conditions $\mathbf{y}(0)=[1,1.5]^{T} \quad \mathbf{y}^{\prime}(0)=\mathbf{0}$. The same system was used in [9] to compare the performance of different $\mathrm{G}(\alpha)$ methods. We compute a reference solution using the MATLAB ode15s solver and we compare the performance of the TR-BDF2 method, of its parent methods, i.e. the off-centered Trapezoidal Rule or $\theta$ - method (setting $\theta=0.51$ ) and the BDF2 method, and of 4 different $\mathrm{G}(\alpha)$ methods, more specifically the Newmark method with $\rho_{\infty}=0,0.25$ and the $\mathrm{CH}-\alpha$ method with the same values of the dissipation parameter. As it can be seen in figure 3 , while the performance of the $\mathrm{G}(\alpha)$ methods and of the TR-BDF2 method is essentially analogous on the the fast and strongly damped variable, the latter is in general the most accurate on the slow variables. 


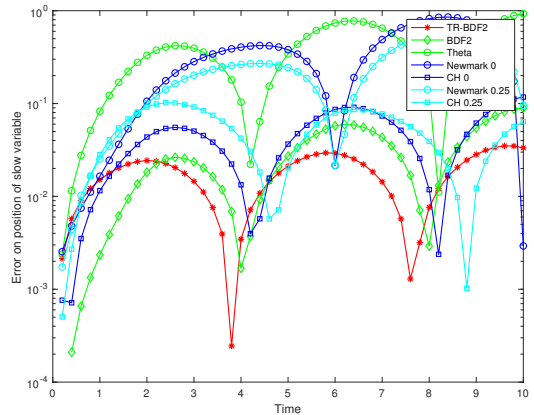

a)

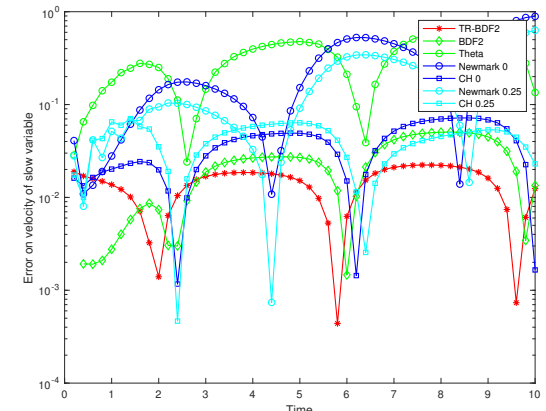

b)

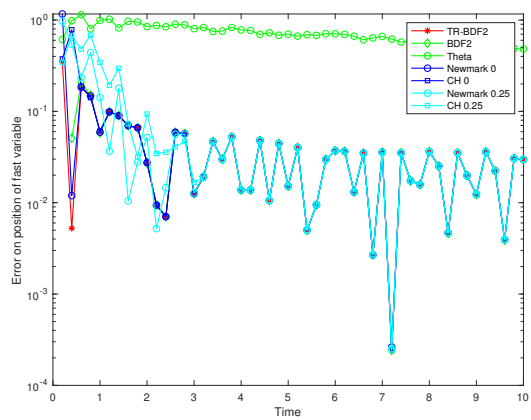

c)

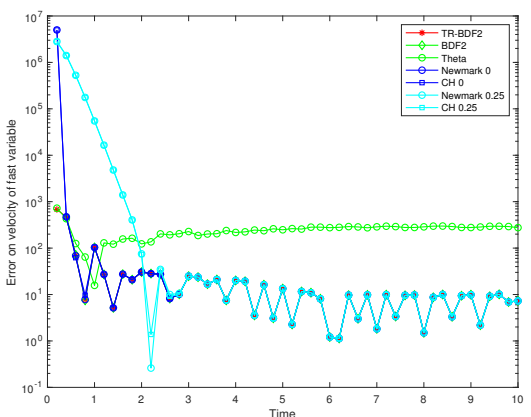

d)

Figure 3: Absolute errors in the test with 2 degrees of freedom on a) position for the slow degree of freedom b) velocity for the slow degrees of freedom c) position for the fast degree of freedom b) velocity for the fast degrees of freedom, as computed by TR-BDF2 and several other methods. 


\subsection{Wave equation in 1 dimension with strongly varying coefficients}

We then consider the one dimensional wave propagation problem described by

$$
\left\{\begin{array}{l}
\rho \frac{\partial^{2} u}{\partial t^{2}}-\frac{\partial}{\partial x}\left(E(x) \frac{\partial u}{\partial x}\right)=0, \quad \text { in }[0, L] \times[0, T] \\
u(0, t)=0, \quad \text { on }[0, T] \\
E(L) \frac{\partial u}{\partial x}(L, T)=0, \quad \text { on }[0, T] \\
u(x, 0)=0, \quad \text { in }[0, L] \\
\frac{\partial u}{\partial t}=-1, \quad \text { in }[0, L] .
\end{array}\right.
$$

These equations model the displacement $u$ of a clamped-free elastic rod with a unit constant cross-sectional area, length $L$, constant density $\rho$ and Young modulus given by $E(x)$. The same problem has also been considered in [17, [23], 9] to study the performance of $\mathrm{G}(\alpha)$ methods in a stiff case in which strong oscillations and numerical overshoots can arise. In particular, we assume $L=10.5, \rho=0.01$ and a Young modulus such that

$$
E(x)= \begin{cases}10^{7} & 0 \leq x<0.5 \\ 10^{2} & 0.5 \leq x<10 \\ 10^{7} & 10 \leq x \leq 10.5\end{cases}
$$

which yields a rather stiff problem. The system is discretized using $\mathbb{P}_{1}$ finite elements on the spatial domain $[0, L]$ and either the TR-BDF2 method, the BDF2 method or the Newmark method with $\beta=1 / 4$, $\gamma_{N}=1 / 2$ for the time discretization. In all cases we have used a mesh with 21 nodes and $\Delta t=0.025 \mathrm{~s}$. As a reference solution, we consider the one obtained by computing the exponential of the matrix of the spatial semi-discretization. In table 1 we report the absolute errors between the considered methods and the reference solution, computed at two different final times. It can be observed that the TR-BDF2 method yields consistently the smallest errors among the three second order methods under comparison. The time series of the displacements and velocities corresponding to the end of the rod $(x=L)$ are reported instead in Figures 4, 5, while the corresponding absolute differences between the computed solutions and the reference one are displayed in Figures 6 and 7 . Again, the smaller error produced by the TR-BDF2 method is apparent. 


\begin{tabular}{|l|l|l|l|l|}
\hline$T$ & Method & Error $L^{\infty}\left(L^{2}\right)$ & Error $L^{2}\left(H^{1}\right)$ & Error $L^{\infty}\left(L^{\infty}\right)$ \\
\hline \hline 1 & BDF2 & $6.63 \mathrm{e}-2$ & 0.59 & $2.50 \mathrm{e}-2$ \\
\hline 1 & Newmark & $2.46 \mathrm{e}-2$ & 0.19 & $6.99 \mathrm{e}-3$ \\
\hline 1 & TR-BDF2 & $1.51 \mathrm{e}-2$ & $6.00 \mathrm{e}-2$ & $2.67 \mathrm{e}-3$ \\
\hline \hline 2.5 & BDF2 & 0.32 & 2.20 & $5.41 \mathrm{e}-2$ \\
\hline 2.5 & Newmark & 0.15 & 1.06 & $2.51 \mathrm{e}-2$ \\
\hline 2.5 & TR-BDF2 & 0.14 & 0.92 & $7.73 \mathrm{e}-3$ \\
\hline \hline
\end{tabular}

Table 1: Absolute errors in the approximation of a stiff 1D wave equation

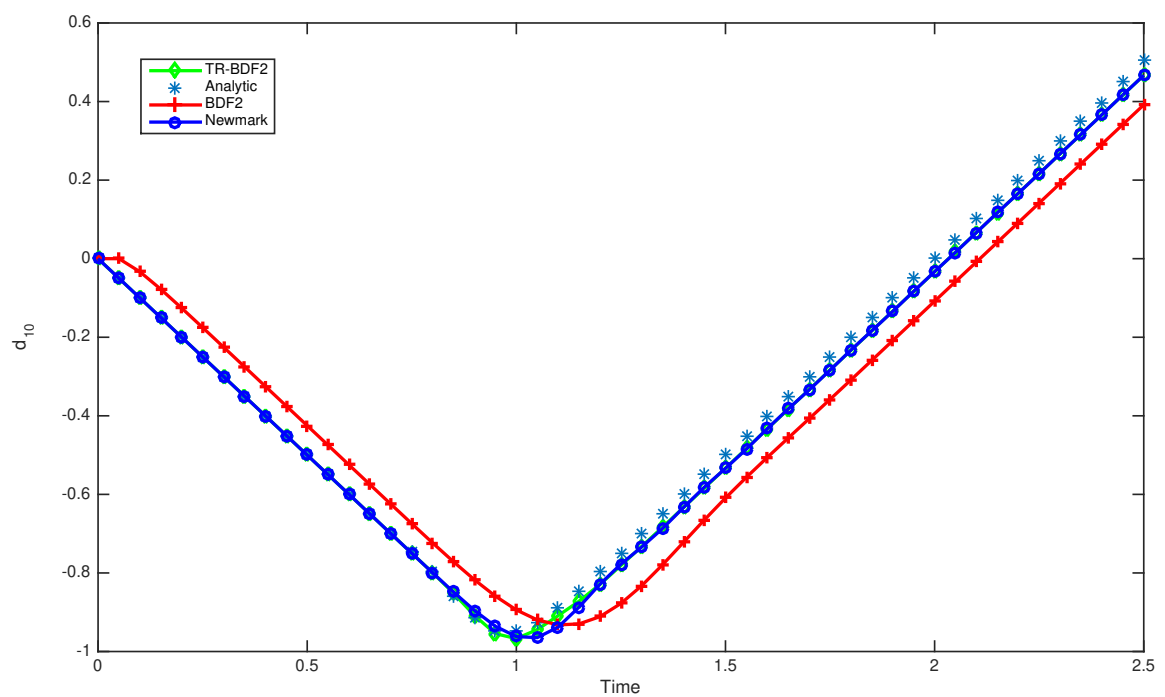

Figure 4: Displacement in $\mathrm{x}=10.5$ 


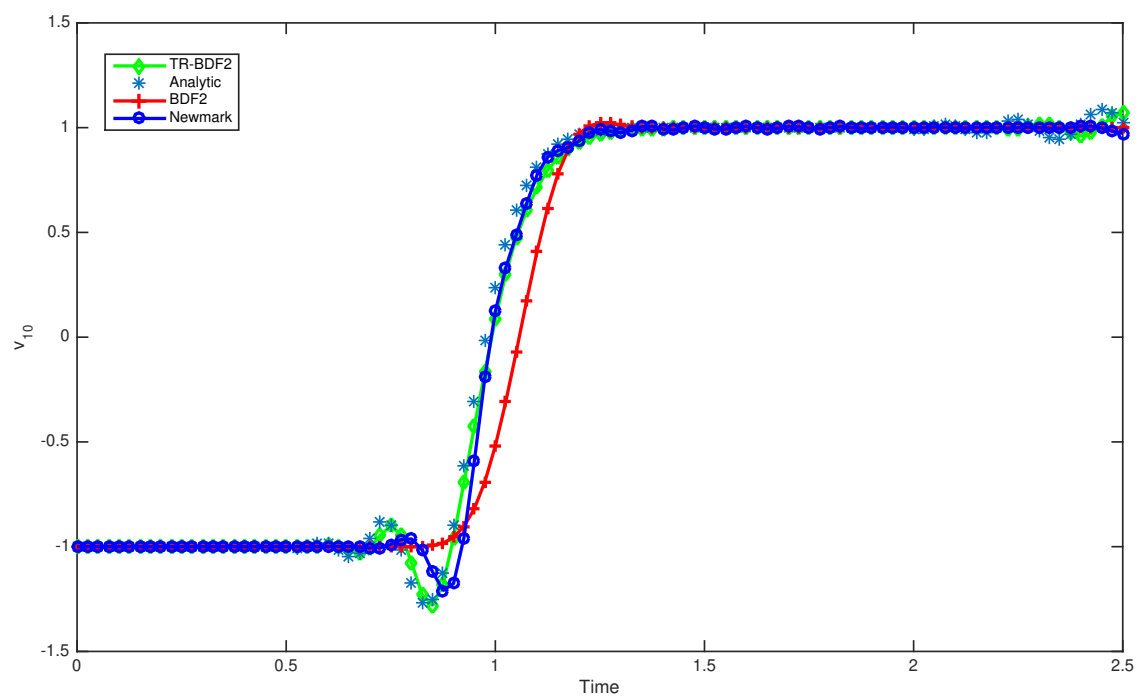

Figure 5: Velocity in $\mathrm{x}=10.5$

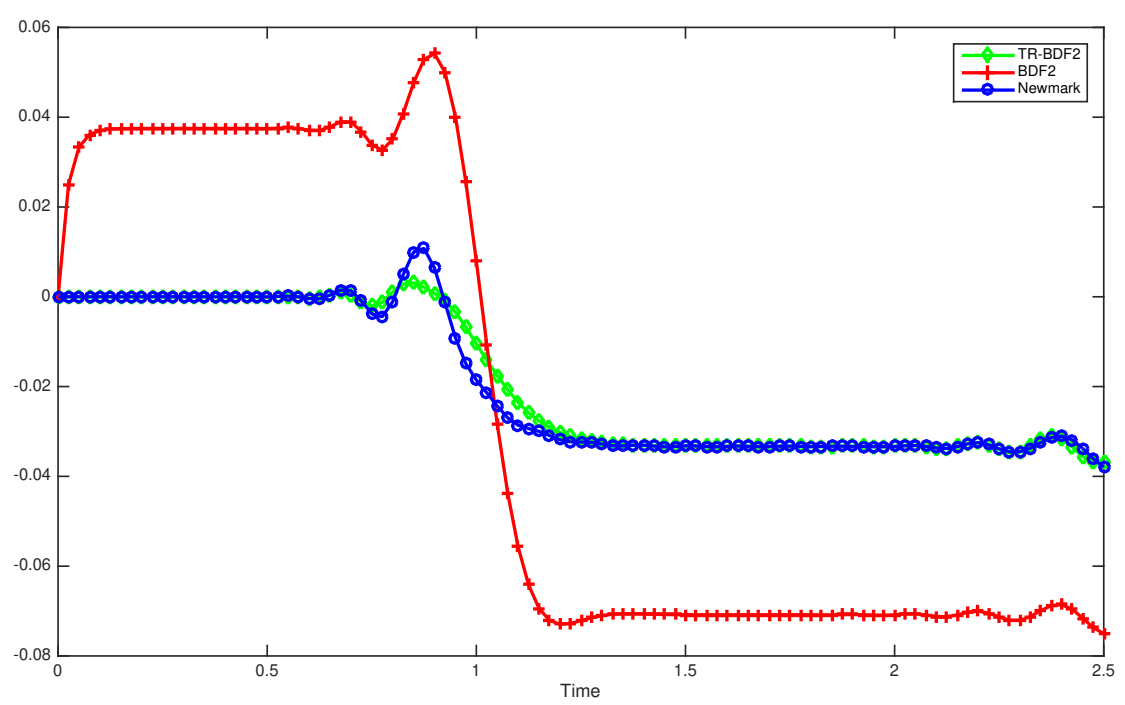

Figure 6: Displacement difference from Analytic solution in $\mathrm{x}=10.5$ 


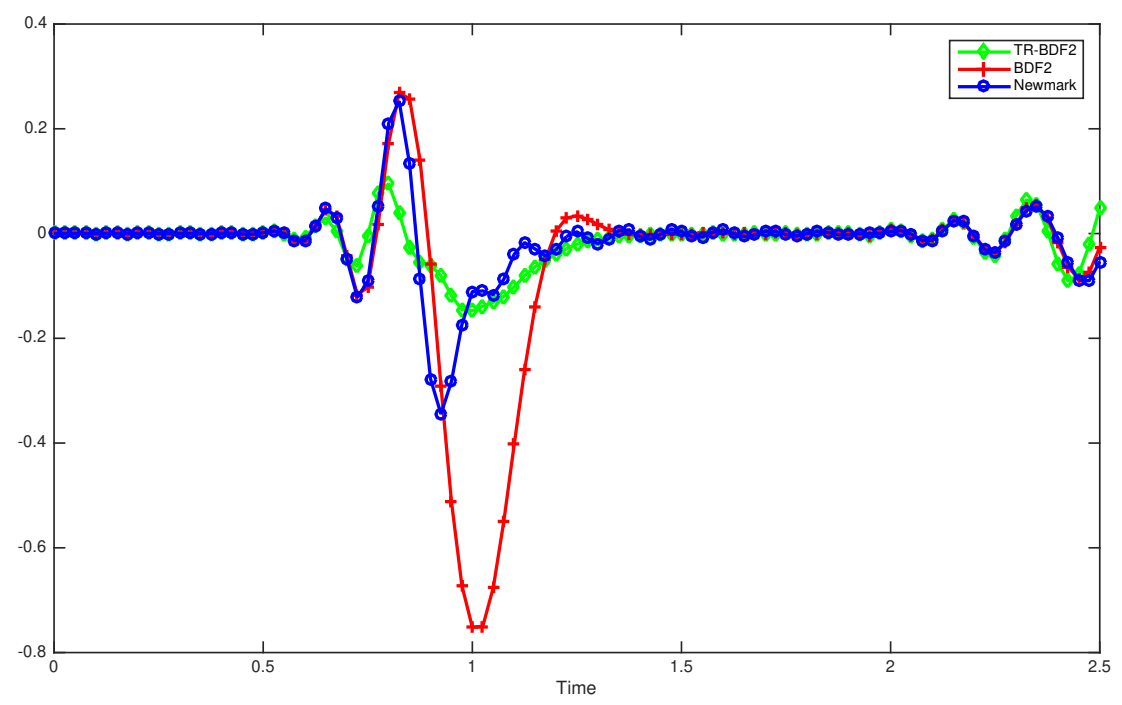

Figure 7: Velocity difference from Analytic solution in $\mathrm{x}=10.5$

\subsection{Two-dimensional test with analytic solu- tion}

In order to verify the implementation used in the more advanced tests presented in Section 4.4, we have considered the the problem

$$
\begin{cases}\frac{\partial^{2} u}{\partial t^{2}}-2 \Delta u=0 & \text { in } \Omega \times[0, T], \\ u(x, y, 0)=0 & \text { in } \Omega, \\ \frac{\partial u}{\partial t}(x, y, 0)=2 \pi \sin (\pi x) \sin (\pi y) & \text { in } \Omega, \\ u(x, y, t)=0 & \text { on } \partial \Omega \times[0, T],\end{cases}
$$

where the domain $\Omega=[0,1] \times[0,1]$ and $T=1$. The solution for this problem is given by $u(x, y, t)=\sin (2 \pi t) \sin (\pi x) \sin (\pi y)$. This solution has been used to perform a convergence study of the TR-BDF2 time discretization, coupled again to a $\mathbb{P}_{1}$ finite element discretization in space. To estimate empirically the convergence order, we choose the maximum diameter $h$ of a mesh element equal in size to the time step $\Delta t$ and we compute the following expression for two different values of $\Delta t$ :

$$
r_{e m p}=\frac{\log \left(\operatorname{err}\left(\Delta t_{2}\right) / \operatorname{err}\left(\Delta t_{1}\right)\right)}{\log \left(\Delta t_{2} / \Delta t_{1}\right)} .
$$

The results of the convergence test are displayed in table 2 .

We have also repeated the computation using the BDF2 method and the Newmark method with $\beta=1 / 4, \gamma_{N}=1 / 2$. The results are 


\begin{tabular}{|l|l|l|l|l|}
\hline$h=\Delta t$ & $L^{\infty}\left(L^{2}\right)$ error & $L^{2}\left(H^{1}\right)$ Error & $r_{e m p}$ in $L^{\infty}\left(L^{2}\right)$ & $r_{e m p}$ in $L^{2}\left(H^{1}\right)$ \\
\hline \hline 0.1 & $1.26 \mathrm{e}-2$ & $4.08 \mathrm{e}-2$ & & \\
\hline 0.05 & $2.78 \mathrm{e}-3$ & $8.79 \mathrm{e}-3$ & 2.17 & 2.21 \\
\hline 0.025 & $6.39 \mathrm{e}-4$ & $2.04 \mathrm{e}-3$ & 2.12 & 2.11 \\
\hline 0.0125 & $1.52 \mathrm{e}-4$ & $4.91 \mathrm{e}-4$ & 2.07 & 2.05 \\
\hline 0.00625 & $3.71 \mathrm{e}-5$ & $1.21 \mathrm{e}-4$ & 2.04 & 2.03 \\
\hline 0.003125 & $9.15 \mathrm{e}-6$ & $2.99 \mathrm{e}-5$ & 2.02 & 2.01 \\
\hline
\end{tabular}

Table 2: Convergence behaviour of the TR-BDF2 method in test (36), absolute errors and convergence rates in $L^{\infty}\left(L^{2}\right)$ and $L^{2}\left(H^{1}\right)$ norms.

shown in table 3 and figure 8 . While second order convergence is achieved by all methods, the results clearly display the significantly smaller errors obtained by the TR-BDF2 method.

\begin{tabular}{|l|l|l|l|}
\hline$h=\Delta t$ & Method & $L^{\infty}\left(L^{2}\right)$ error & $L^{2}\left(H^{1}\right)$ error \\
\hline \hline 0.025 & BDF2 & $1.93 \mathrm{e}-2$ & $5.58 \mathrm{e}-2$ \\
\hline 0.025 & Newmark & $3.35 \mathrm{e}-3$ & $8.22 \mathrm{e}-3$ \\
\hline 0.025 & TR-BDF2 & $6.39 \mathrm{e}-4$ & $2.04 \mathrm{e}-3$ \\
\hline \hline 0.0125 & BDF2 & $4.87 \mathrm{e}-3$ & $1.38 \mathrm{e}-2$ \\
\hline 0.0125 & Newmark & $8.32 \mathrm{e}-4$ & $1.96 \mathrm{e}-3$ \\
\hline 0.0125 & TR-BDF2 & $1.52 \mathrm{e}-4$ & $4.91 \mathrm{e}-4$ \\
\hline \hline 0.00625 & BDF2 & $1.22 \mathrm{e}-4$ & $3.44 \mathrm{e}-3$ \\
\hline 0.00625 & Newmark & $2.06 \mathrm{e}-4$ & $4.78 \mathrm{e}-4$ \\
\hline 0.00625 & TR-BDF2 & $3.71 \mathrm{e}-5$ & $1.2 \mathrm{e}-4$ \\
\hline
\end{tabular}

Table 3: Convergence behaviour of the BDF2, Newmark and TR-BDF2 method in test (36), absolute errors in $L^{\infty}\left(L^{2}\right)$ and $L^{2}\left(H^{1}\right)$ norms. 

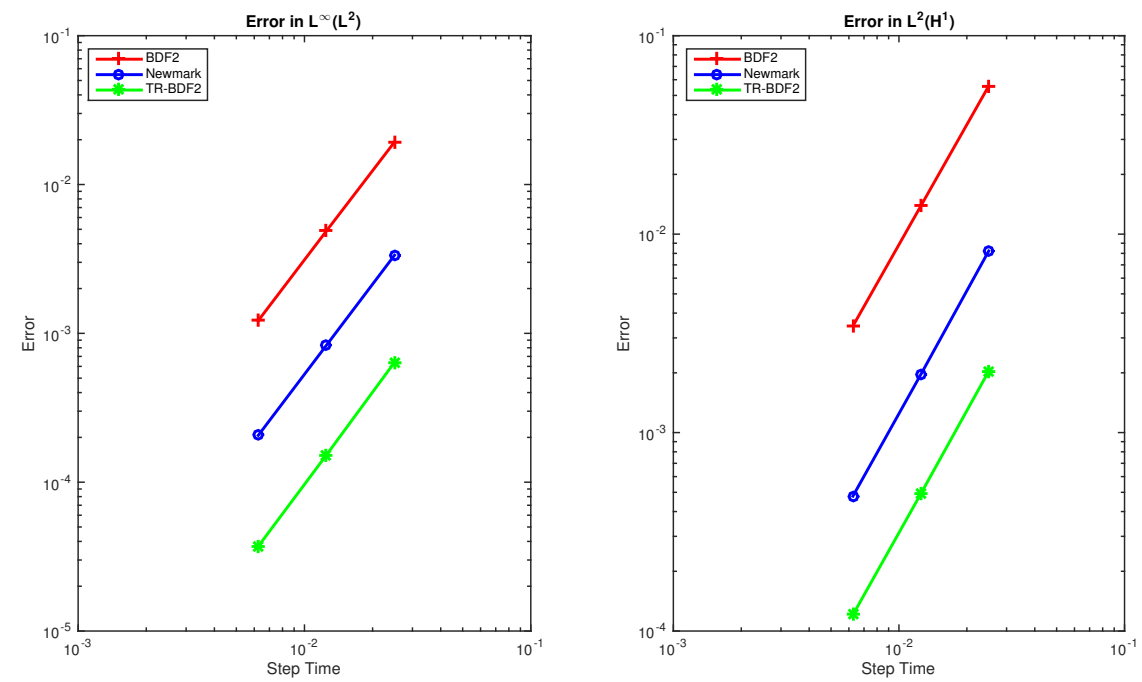

Figure 8: Comparison of convergence behaviour of different methods in test (36).

\subsection{Two dimensional elasticity}

We consider the plane-strain equations of two dimensional elasticity, presented in [19] as the first order system:

$$
\begin{aligned}
\frac{\partial \sigma^{x, x}}{\partial t} & =(\lambda+2 \mu) \frac{\partial u}{\partial x}+\lambda \frac{\partial v}{\partial y} \\
\frac{\partial \sigma^{x, y}}{\partial t} & =\mu \frac{\partial v}{\partial x}+\mu \frac{\partial u}{\partial y} \\
\frac{\partial \sigma^{y, y}}{\partial t} & =\lambda \frac{\partial u}{\partial x}+(\lambda+2 \mu) \frac{\partial v}{\partial y} \\
\rho \frac{\partial u}{\partial t} & =\frac{\partial \sigma^{x, x}}{\partial x}+\frac{\partial \sigma^{x, y}}{\partial y} \\
\rho \frac{\partial v}{\partial t} & =\frac{\partial \sigma^{y, x}}{\partial x}+\frac{\partial \sigma^{y, y}}{\partial y} .
\end{aligned}
$$

Here, $\sigma^{i, j}$ denote the components of the (symmetric) stress tensor $\boldsymbol{\Sigma}$, $\mathbf{u}=(u, v)$ is the velocity vector with components $u, v$ in the $x, y$ directions, respectively, $\rho$ is the medium density and $\lambda, \mu$ are the medium Lamé coefficients. For the purpose of our work, we reformulate these equations as second order equations in terms of the displacements $\boldsymbol{\delta}=\left(\delta^{x}, \delta^{y}\right)$. As a result, equations (38) are equivalent to 


$$
\begin{aligned}
\sigma^{x, x} & =(\lambda+2 \mu) \frac{\partial \delta^{x}}{\partial x}+\lambda \frac{\partial \delta^{y}}{\partial y} \\
\sigma^{x, y} & =\mu \frac{\partial \delta^{y}}{\partial x}+\mu \frac{\partial \delta^{x}}{\partial y} \\
\sigma^{y, y} & =\lambda \frac{\partial \delta^{x}}{\partial x}+(\lambda+2 \mu) \frac{\partial \delta^{x}}{\partial y} \\
\rho \frac{\partial^{2} \delta^{x}}{\partial t^{2}} & =\frac{\partial \sigma^{x, x}}{\partial x}+\frac{\partial \sigma^{x, y}}{\partial y} \\
\rho \frac{\partial^{2} \delta^{y}}{\partial t^{2}} & =\frac{\partial \sigma^{y, x}}{\partial x}+\frac{\partial \sigma^{y, y}}{\partial y},
\end{aligned}
$$

which, after substitution of the expression for the stresses into the momentum equation, can be rewritten in vector form as

$$
\rho \frac{\partial^{2} \boldsymbol{\delta}}{\partial t^{2}}=\operatorname{div} \boldsymbol{\Sigma}=\mu \Delta \boldsymbol{\delta}+(\lambda+\mu) \nabla \operatorname{div} \boldsymbol{\delta} .
$$

As discussed in [19, this system has $\mathrm{P}$ and $\mathrm{S}$-wave solutions with propagation speeds

$$
c_{P}=\sqrt{\frac{\lambda+2 \mu}{\rho}} \quad c_{s}=\sqrt{\frac{\mu}{\rho}} .
$$

We consider the above equations on the domain $\Omega=[0,3] \times[0,3]$ and on the time interval $[0, T]$, with $T=10^{-2} \mathrm{~s}$. We assume that the density is constant and equal one in all the domain, while the elastic constants are such that a more rigid inclusion is embedded as shown in Figure 9. More specifically, in the darker region, denoted as Zone 2 in Figure $9 \mathrm{~b}$ ), whose boundary is described by the equation is $\left(x-x_{0}\right)^{2}+\left(y-y_{0}\right)^{2}=1 / 100$, with $\left(x_{0}, y_{0}\right)=(1.65,1.65)$, we assume that $\lambda=200, \mu=100$, while in the region represented in red one has $\lambda=2, \mu=1$. We then proceed to study the wave propagation resulting from imposing at the initial time a displacement in the region denoted as Zone 1 in Figure 9b), whose boundary is described by the equation $\left(x-x_{0}\right)^{2}+\left(y-y_{0}\right)^{2}=9 / 10^{4}$. More specifically, we consider the function defined as $d(x, y)=\exp \left(-\left(x-x_{0}\right)^{2} / 2-\left(y-y_{0}\right)^{2} / 2\right)$ in Zone 1 and $d(x, y)=0$ in the rest of the domain and we take as initial conditions

$$
\delta^{x}=0, \delta^{y}=d(x, y), \frac{\partial \delta^{x}}{\partial t}=0, \frac{\partial \delta^{y}}{\partial t}=v,
$$

where $v$ is defined by the finite difference approximation $v(x, y)=$ $d(x, y) / \Delta t$, thus corresponding to the velocity that would be obtained if the impulsive displacement $d(x, y)$ had been produced in a time 

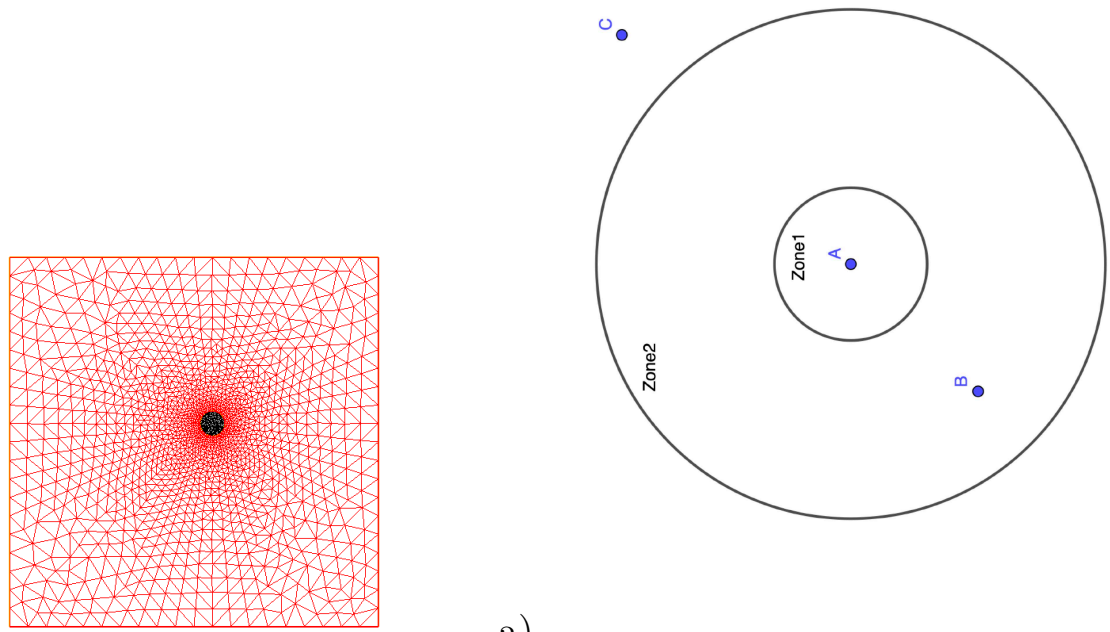

a)

b)

Figure 9: Sketch of a) the computational domain and mesh and b) the areas with more rigid material and initial perturbations.

interval of size $\Delta t$ starting from zero. For the numerical discretization we consider $\mathbb{P}_{1}$ continuous finite elements on the locally refined unstructured mesh depicted in Figure 9 a), which is composed of 15036 vertices and 7559 elements with an average diameter equal to $h=2.39 \times 10^{-2}$ in Zone 2 and equal to $h=0.22$ outside. For the time discretization we use a time step of $\Delta t=1.25 \times 10^{-4}$, which entails a Courant number based on the $\mathrm{P}$-wave speed of $c_{P} \Delta t / h \approx 1.04$ in Zone 1 and $c_{P} \Delta t / h \approx 0.01$ in the rest of the domain, thus mimicking a situation in which an unconditionally stable method is typically applied.

Since an analytic solution is not available, we consider as a reference solution that computed by the same space discretization coupled to a fully implicit Gauss method of order four, see e.g. [11. Therefore, the errors with respect to the reference solution are an estimate of the time discretization error only. We compare the implicit Euler method, the Crank-Nicolson method, the Newmark method with $\beta=1 / 3$ and $\gamma_{N}=1 / 2$ and the TR-BDF2 method. We include also the implicit Euler method as a representative of a robust, unconditionally stable, albeit first order method. A pictorial view of the resulting wave 
propagation on the portion of the domain around Zone 2 at different instants as computed by the TR-BDF2 method is reported in Figures 1011 . 


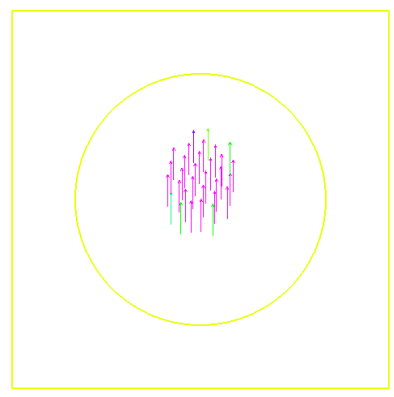

a)
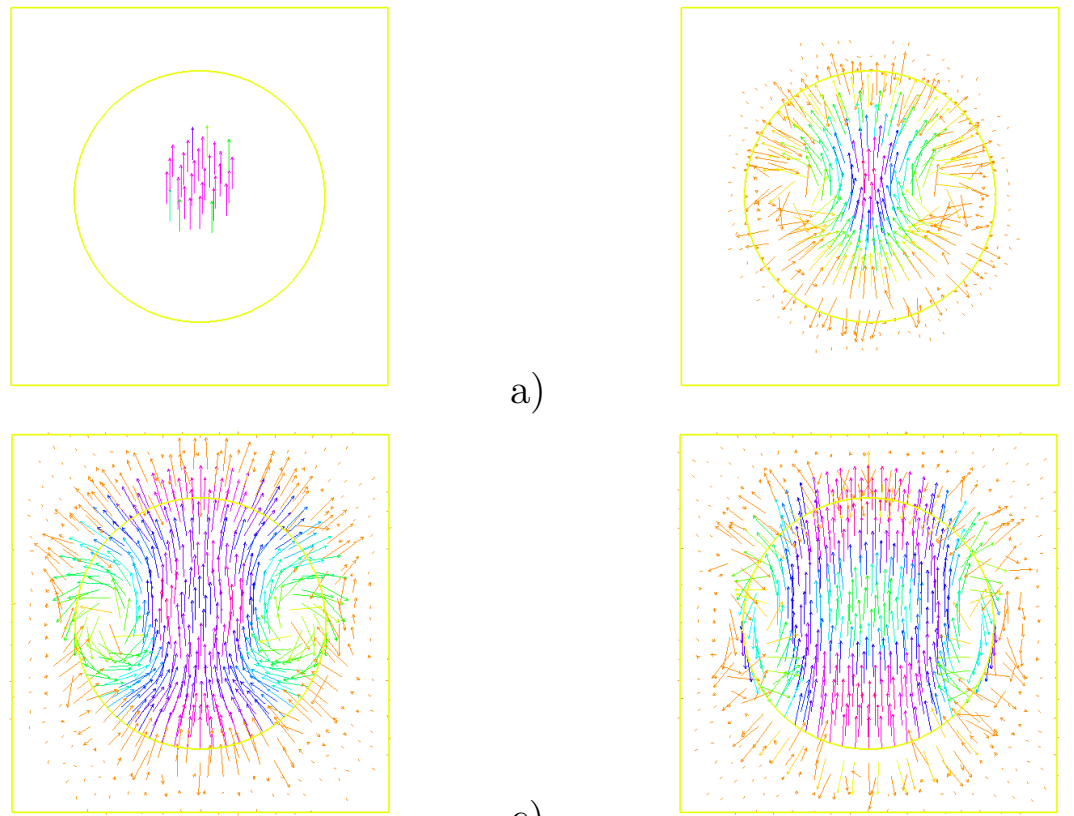

b)

c)

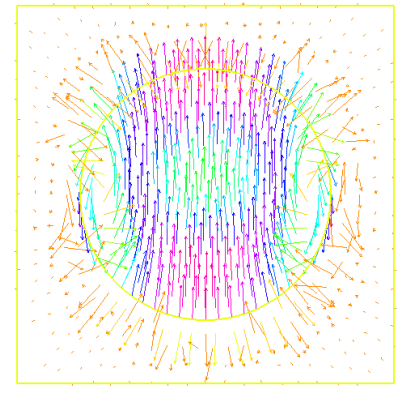

d)

Figure 10: Displacement vector field at times a) $t=0$, b) $t=2.5 \times 10^{-3}$, c) $t=5 \times 10^{-3}$, d) $t=7.5 \times 10^{-3}$.

The global relative errors with respect to the reference solution are then reported in Tables 4 and 5 for the two displacement components, respectively. It can be observed that the TR-BDF2 consistently yields the smallest error values. The behaviour of the computed solutions

\begin{tabular}{||l|l|l|}
\hline Method & Error $L^{\infty}\left(L^{2}\right)$ & Error $L^{2}\left(H^{1}\right)$ \\
\hline \hline Implicit Euler & 0.69 & $2.64 \mathrm{e}-2$ \\
\hline Newmark & 0.19 & $1.85 \mathrm{e}-3$ \\
\hline Crank-Nicolson & $8.21 \mathrm{e}-2$ & $1.15 \mathrm{e}-3$ \\
\hline TR-BDF2 & $4.40 \mathrm{e}-2$ & $5.73 \mathrm{e}-4$ \\
\hline
\end{tabular}

Table 4: Relative errors on the displacement in the $x$-direction

over the whole time interval $[0, T]$ is also displayed in Figure 12 at the three control points A,B and $\mathrm{C}$ shown in Figure 9 b). Point A point is located at the center of Zone 1, where the initial displacement occurs, point $\mathrm{B}$ is in Zone 2 and $\mathrm{C}$ is the outer region but close to Zone 2 . It can be observed that, apart from the solutions computed by the implicit Euler method, the others are all in good agreement. The 

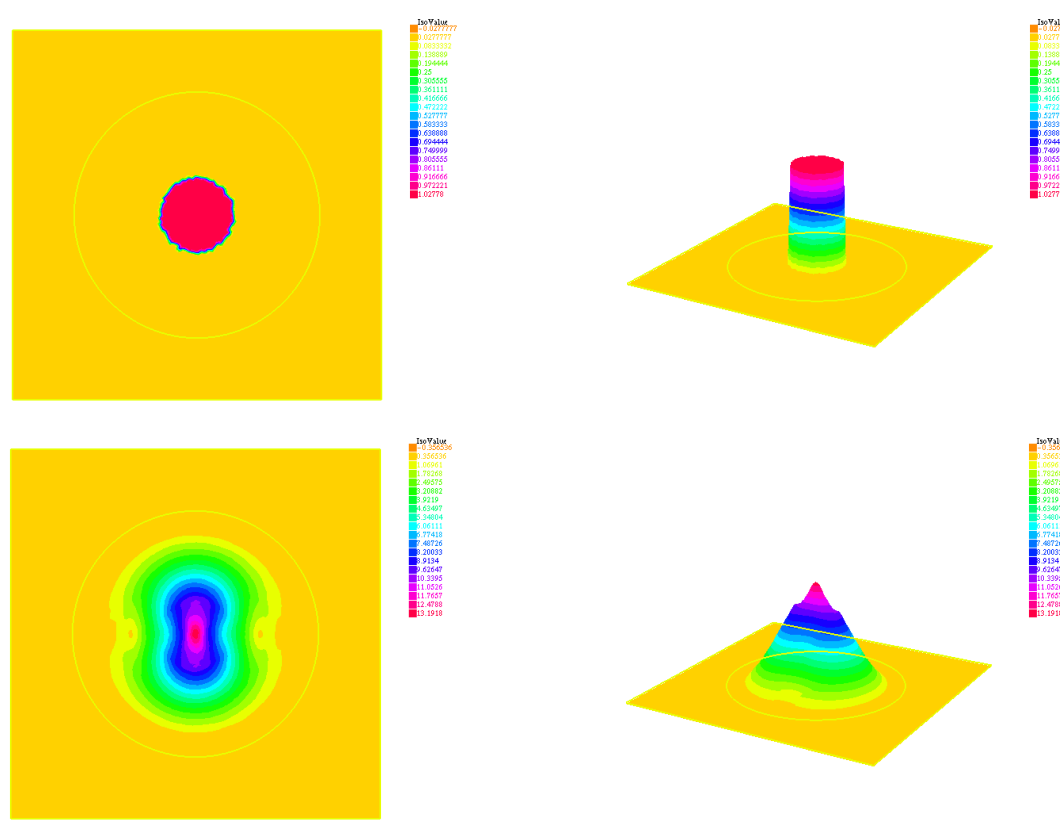

a)

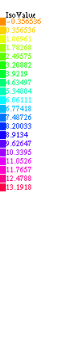

b)
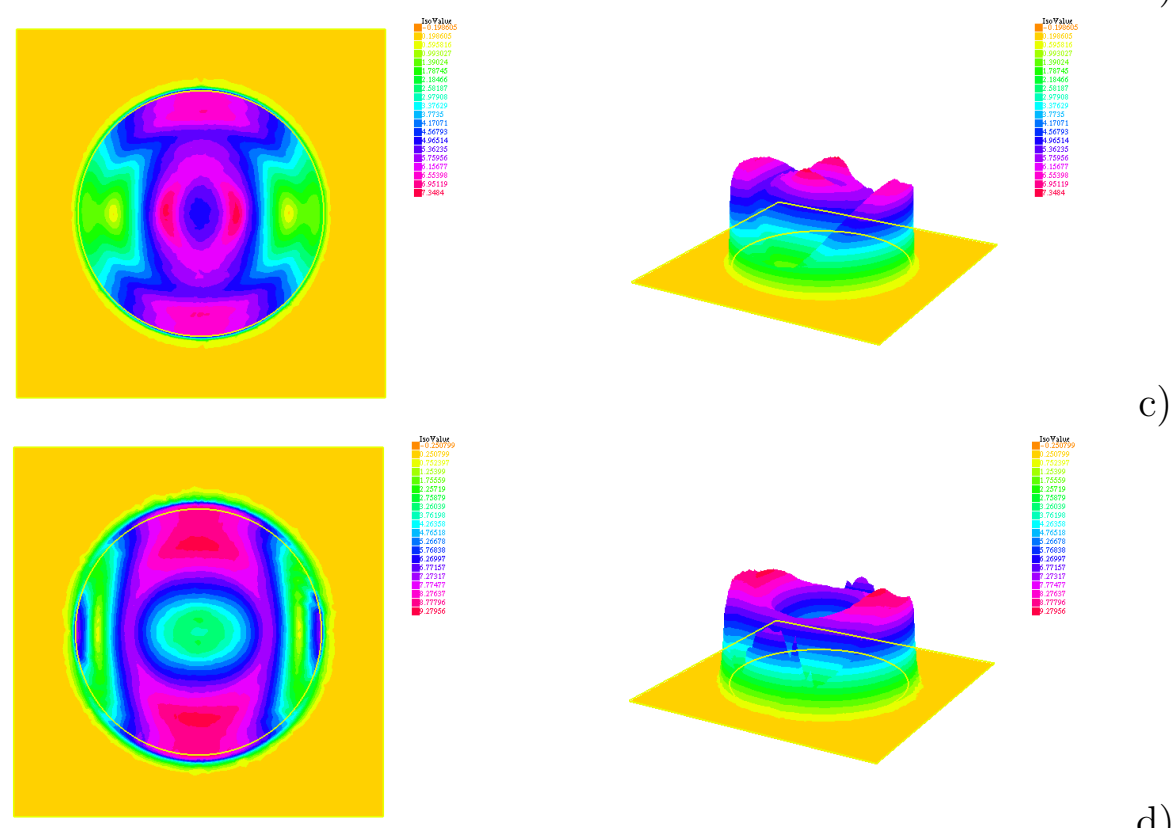

d)

Figure 11: Absolute value of the displacement vector field at times a) $t=0$, b) $t=2.5 \times 10^{-3}$, c) $t=5 \times 10^{-3}$, d) $t=7.5 \times 10^{-3}$. 


\begin{tabular}{||l|l|l|}
\hline Method & Error $L^{\infty}\left(L^{2}\right)$ & Error $L^{2}\left(H^{1}\right)$ \\
\hline \hline Euler & $7.07 \mathrm{e}-2$ & $1.71 \mathrm{e}-2$ \\
\hline Newmark & $8.35 \mathrm{e}-3$ & $5.29 \mathrm{e}-3$ \\
\hline Crank-Nicolson & $5.89 \mathrm{e}-3$ & $3.68 \mathrm{e}-4$ \\
\hline TR-BDF2 & $2.93 \mathrm{e}-3$ & $1.82 \mathrm{e}-4$ \\
\hline
\end{tabular}

Table 5: Relative errors on the displacement in the $y$-direction

corresponding absolute errors with respect to the reference solution are shown in Figure 13, while the error norms at the three control points are reported in Tables 6, 7 and 8, respectively. These results highlight the fact that the TR-BDF2 method yields more accurate solutions both in the higher and lower Courant number regions, with errors that are consistently at least $50 \%$ smaller than those of the other methods. This is especially important in view to the combination of these methods with higher order discretizations in space. 

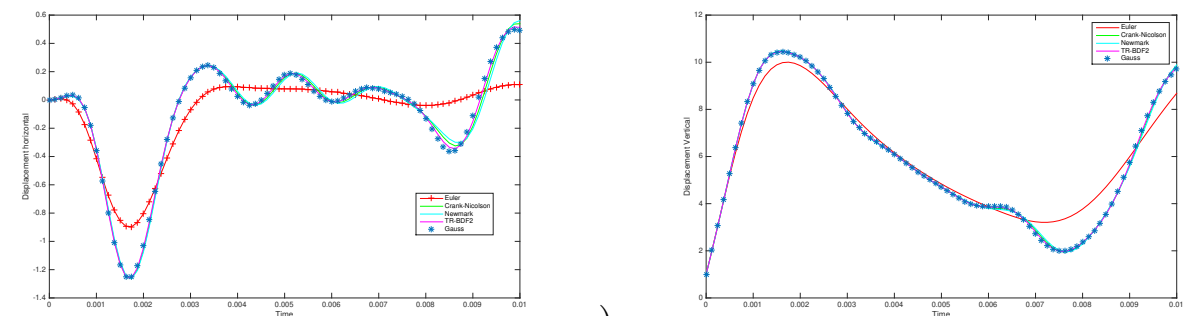

a)

b)

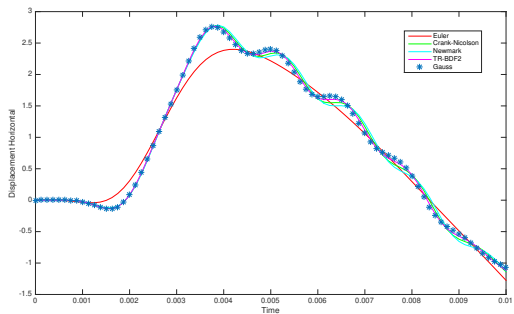

c)

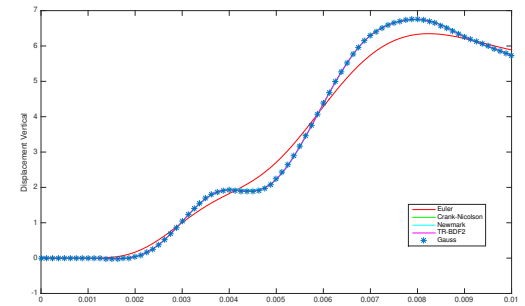

d)

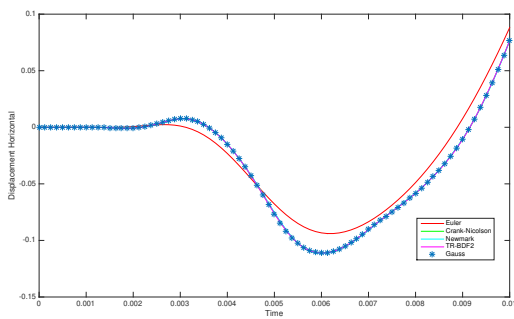

e)

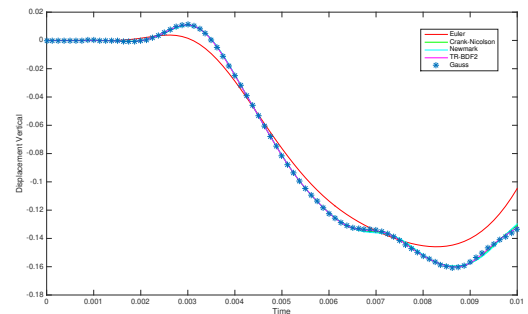

f)

Figure 12: Left column: displacement in the $x$-direction, right column: displacement in the $y$-direction, computed in a)-b) Point A, c)-d) Point B, e)-f) Point C. 


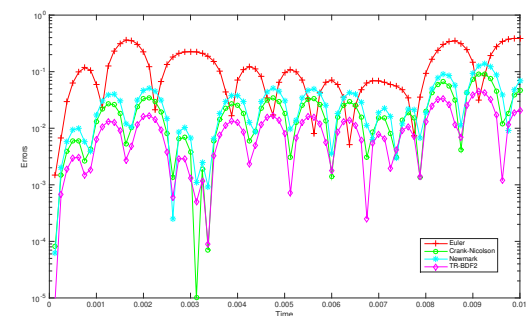

a)

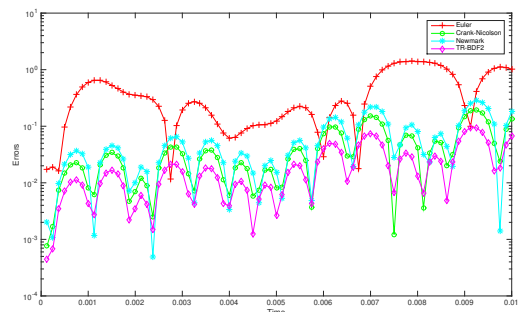

b)

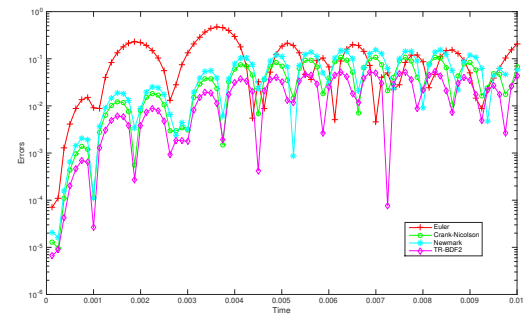

c)

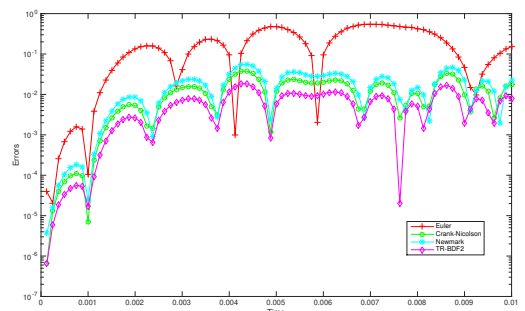

d)

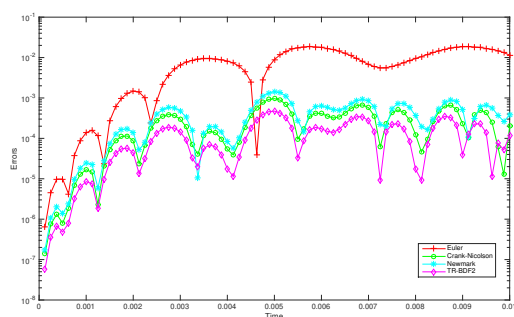

e)

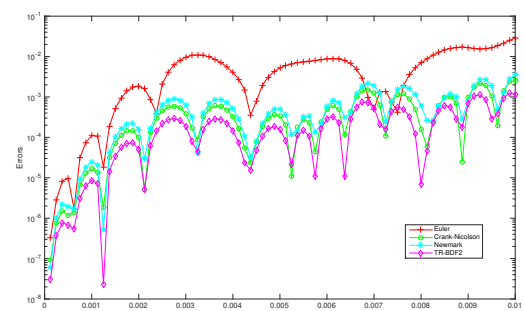

f)

Figure 13: Left column: absolute error on the displacement in the $x$-direction, right column: absolute error on the displacement in the $y$-direction, computed in a)-b) Point A, c)-d) Point B, e)-f) Point C. 


\begin{tabular}{|l|c|c|c|c|}
\hline Method & $L^{2}$ error on $\delta^{x}$ & $L^{\infty}$ error on $\delta^{x}$ & $L^{2}$ error on $\delta^{y}$ & $L^{\infty}$ error on $\delta^{y}$ \\
\hline \hline Implicit Euler & $1.74 \mathrm{e}-2$ & 0.39 & $6.31 \mathrm{e}-2$ & 1.41 \\
\hline Newmark & $4.32 \mathrm{e}-3$ & 0.137 & $9.39 \mathrm{e}-3$ & 0.28 \\
\hline Crank-Nicolson & $2.99 \mathrm{e}-3$ & $9.16 \mathrm{e}-2$ & $6.50 \mathrm{e}-3$ & 0.19 \\
\hline TR-BDF2 & $1.46 \mathrm{e}-3$ & $4.52 \mathrm{e}-2$ & $3.21 \mathrm{e}-3$ & $9.43 \mathrm{e}-2$ \\
\hline \hline
\end{tabular}

Table 6: Absolute errors on displacement in Point A

\begin{tabular}{|l|c|c|c|c|}
\hline Method & $L^{2}$ error on $\delta^{x}$ & $L^{\infty}$ error on $\delta^{x}$ & $L^{2}$ error on $\delta^{y}$ & $L^{\infty}$ error on $\delta^{y}$ \\
\hline \hline Implicit Euler & $1.58 \mathrm{e}-2$ & 0.47 & $2.74 \mathrm{e}-2$ & 0.55 \\
\hline Newmark & $7.50 \mathrm{e}-3$ & 0.15 & $2.31 \mathrm{e}-3$ & $5.62 \mathrm{e}-2$ \\
\hline Crank-Nicolson & $5.29 \mathrm{e}-3$ & 0.11 & $1.58 \mathrm{e}-3$ & $3.76 \mathrm{e}-2$ \\
\hline TR-BDF2 & $2.64 \mathrm{e}-3$ & $5.36 \mathrm{e}-2$ & $7.79 \mathrm{e}-4$ & $1.83 \mathrm{e}-2$ \\
\hline \hline
\end{tabular}

Table 7: Absolute errors on displacement in Point B

\begin{tabular}{|l|c|c|c|c|}
\hline Method & $L^{2}$ error on $\delta^{x}$ & $L^{\infty}$ error on $\delta^{x}$ & $L^{2}$ error on $\delta^{y}$ & $L^{\infty}$ error on $\delta^{y}$ \\
\hline \hline Implicit Euler & $1.04 \mathrm{e}-3$ & $1.86 \mathrm{e}-2$ & $9.14 \mathrm{e}-4$ & $2.86 \mathrm{e}-2$ \\
\hline Newmark & $5.36 \mathrm{e}-5$ & $1.43 \mathrm{e}-3$ & $1.03 \mathrm{e}-4$ & $3.63 \mathrm{e}-3$ \\
\hline Crank-Nicolson & $3.65 \mathrm{e}-5$ & $9.63 \mathrm{e}-4$ & $7.68 \mathrm{e}-5$ & $2.60 \mathrm{e}-3$ \\
\hline TR-BDF2 & $1.79 \mathrm{e}-5$ & $4.72 \mathrm{e}-4$ & $3.96 \mathrm{e}-5$ & $1.24 \mathrm{e}-3$ \\
\hline \hline
\end{tabular}

Table 8: Absolute errors on displacement in Point C

\section{Conclusions and future work}

We have proposed a reformulation of the TR-BDF2 method that allows to apply it without overheads in terms of computational cost or memory to large scale problems in structural mechanics. Our work extends the analysis and the comparison of [22] and a similar reformulation proposed for two-stage Rosenbrock methods in [23]. In particular, we have presented a reformulation of this method that only implies the solution of nonlinear systems of the same size as the number of degrees of freedom necessary to describe the displacement variables. Furthermore, the velocity degrees of freedom do not have to 
be stored explicitly and can be recomputed whenever needed, thus avoiding the shortcomings of naive implementations of solvers for first order ODE systems. An analysis of the dissipative behaviour of the method, which was carried out considering also the damping term, shows that TR-BDF2 is superior in terms of accuracy and efficiency to the classical $\mathrm{G}(\alpha)$ methods. This finding is confirmed by a number of numerical experiments on significant benchmarks of increasing complexity, in which the TR-BDF2 method consistently yields errors least $50 \%$ smaller than those of the other methods, both in high and low Courant number regions. This is especially important in view to the combination of robust, unconditionally stable time discretization methods with higher order discretizations in space.

The future developments of this work include the development of a multirate version of the proposed method, based on the self-adjusting multirate technique described in [3], for application to structural mechanics problems with multiple time scales [10] and to monolithic treatment of fluid structure interaction problems [15, as well as the coupling of the proposed time discretization to high order, adaptive discontinuous finite element spatial discretizations, so as to achieve the performance improvements demonstrated in [25] also in the wave propagation problems typical of structural and seismic engineering.

\section{Acknowledgements}

L.B. would like to thank T. Chacón Rebollo, E. Fernández Nieto and G. Narbona Reina for supporting several visits to Sevilla University, which have allowed the inception and completion of this work. This work has been supported by the Spanish Government Project RTI2018-093521-B-C31.

\section{References}

[1] R.E. Bank, W.M. Coughran, W. Fichtner, E.H. Grosse, D.J. Rose, and R.K. Smith. Transient Simulation of Silicon Devices and Circuits. IEEE Transactions on Electron Devices, 32:19922007, 1985.

[2] K.J. Bathe and E. L.Wilson. Stability and accuracy analysis of direct integration methods. Earthquake Engineering and Structural Dynamics, 1:283-291, 1973.

[3] L. Bonaventura, F. Casella, L. Delpopolo Carciopolo, and A. Ranade. A self adjusting multirate algorithm for robust time 
discretization of partial differential equations. Computers and Mathematics with Applications, 79:2086-2098, 2020.

[4] L. Bonaventura and A. Della Rocca. Unconditionally strong stability preserving extensions of the TR-BDF2 method. Journal of Scientific Computing, 70:859-895, 2017.

[5] O.S. Bursi, A. Gonzalez-Buelga, L. Vulcan, S.A. Neild, and D.J. Wagg. Novel coupling Rosenbrock-based algorithms for real-time dynamic substructure testing. Earthquake Engineering \&6 Structural Dynamics, 37:339-360, 2008.

[6] O.S. Bursi, C. Jua, L. Vulcan, S.A. Neild, and D.J. Wagg. Rosenbrock-based algorithms and subcycling strategies for realtime nonlinear substructure testing. Earthquake Engineering 85 Structural Dynamics, 40:1-19, 2011.

[7] J. Chung and G.M. Hulbert. A time integration algorithm for structural dynamics with improved numerical dissipation: the generalized- $\alpha$ method. Journal of Applied Mechanics, 60:371375, 1993.

[8] J.D. Edwards, J.E. Morel, and D.A. Knoll. Nonlinear variants of the TR/BDF2 method for thermal radiative diffusion. Journal of Computational Physics, 230:1198-1214, 2011.

[9] S. Erlicher, L. Bonaventura, and O. S. Bursi. The analysis of the generalized- $\alpha$ method for non-linear dynamic problems. Computational Mechanics, 28:83-104, 2002.

[10] A. Gravouil and A. Combescure. Multi-time-step explicitimplicit method for non-linear structural dynamics. International Journal for Numerical Methods in Engineering, 50:199-225, 2001.

[11] E. Hairer, H.P. Norsett, and G. Wanner. Solving Ordinary Differential Equations. I Nonstiff Problems (2nd. Revised Edition). Springer-Verlag, 1993.

[12] A.W. Hamkar, S. Hartmann, and J. Rang. A stiffly accurate Rosenbrock-type method of order 2 applied to FE-analyses in finite strain viscoelasticity. Applied Numerical Mathematics, 62:1837-1848, 2012.

[13] S. Hartmann and J. Wensch. Finite element analysis of viscoelastic structures using Rosenbrock-type methods. Computational Mechanics, 40:383-398, 2007.

[14] F. Hecht. New development in freefem++. Journal of Numerical Mathematics, 20:251-265, 2012. 
[15] M. Heil, A.L. Hazel, and J. Boyle. Solvers for large-displacement fluid-structure interaction problems: segregated versus monolithic approaches. Computational Mechanics, 43:91-101, 2008.

[16] M.E. Hosea and L.F. Shampine. Analysis and implementation of TR-BDF2. Applied Numerical Mathematics, 20:21-37, 1996.

[17] T. Hughes and W.Liu. Implicit-explicit finite elements in transient analysis: implementation and numerical examples. Journal of Applied Mechanics, Transactions ASME, 45(2):375-378, 1978.

[18] C.P. Lamarche, A. Bonelli, O.S. Bursi, and R. Tremblay. A Rosenbrock-W method for real-time dynamic substructuring and pseudo-dynamic testing. Earthquake Engineering \& Structural Dynamics, 38:1071-1092, 2009.

[19] R.J. LeVeque. Finite Volume Methods for Hyperbolic Problems. Cambridge University Press, 2004.

[20] J.P. Meijaard. Application of Runge-Kutta-Rosenbrock methods to the analysis of flexible multibody systems. Multibody System Dynamics, 10:263-288, 2003.

[21] N. M. Newmark. A method of computation for structural dynamics. Journal of the Engineering Mechanics Division, 85:67-94, 1959 .

[22] B. Owren and H. H. Simonsen. Alternative integration methods for problems in structural dynamics. Computer Methods in Applied Mechanics and Engineering, 122:1-10, 1995.

[23] R. Piché. An L-stable Rosenbrock method for step-by-step time integration in structural dynamics. Computer Methods in Applied Mechanics and Engineering, 126:343-354, 1995.

[24] G. Tumolo. A mass conservative TR-BDF2 semi-implicit semiLagrangian DG discretization of the shallow water equations on general structured meshes of quadrilaterals. Communications in Applied and Industrial Mathematics, 7:165-190, 2016.

[25] G. Tumolo and L. Bonaventura. A semi-implicit, semiLagrangian, DG framework for adaptive numerical weather prediction. Quarterly Journal of the Royal Meteorological Society, 141:2582-2601, 2015. 